Article

\title{
The Technique of Quadruple Fixed Points for Solving Functional Integral Equations under a Measure of Noncompactness
}

\author{
Hasanen A. Hammad * (1) and Amal A. Khalil \\ Department of Mathematics, Faculty of Science, Sohag University, Sohag 82524, Egypt; \\ amalaboelwafa@science.sohag.edu.eg \\ * Correspondence: hassanein_hamad@science.sohag.edu.eg
}

Received: 27 October 2020; Accepted: 23 November 2020; Published: 28 November 2020

check for updates

\begin{abstract}
Under the idea of a measure of noncompactness, some fixed point results are proposed and a generalization of Darbo's fixed point theorem is given in this manuscript. Furthermore, some novel quadruple fixed points results via a measure of noncompactness for a general class of functions are presented. Ultimately, the solutions to a system of non-linear functional integral equations by the fixed point results obtained are discussed, and non-trivial examples to illustrate the validity of our study are derived.
\end{abstract}

Keywords: a measure of noncompactness; quadruple fixed points; functional integral equations; Darbo's fixed point theorem

MSC: 47H09; 47H10; 34A12

\section{Introduction}

In the nonlinear analysis field, the fixed point technique is used to solve many mathematical problems as it is involved in differential and integral equations, integro-differential equations, fractional calculus, and other disciplines of science and technology; see [1-7].

In the finite-dimensional spaces, this technique was created by Brouwer [8] and is known as "Brouwer's fixed point theorem (FPT)". In 1922, the existence and uniqueness of this technique were studied in a contraction mapping via a complete metric space, known as the "Banach contraction principle [9]". From this moment, the technique acquired special elegance and entered many important applications. For infinite-dimensional spaces under the stipulation of compactness on a set and equivalently on the operator, the results of Brouwer were generalized by Schauder [10].

A measure of noncompactness (MNC) is a very important tool in non-linear functional analysis, especially when dealing with metric and topological fixed point theory. In 1930, Kuratowski first introduced an MNC in [11] as follows:

$$
\varrho(\Xi)=\inf \left\{\omega>0: \Xi \subset \cup_{l=1}^{m} \Xi_{l} \text { with } \operatorname{diam}\left(\Xi_{l}\right) \leq \omega, 1 \leq l \leq m<\infty\right\},
$$

where $\Xi_{l}$ is a bounded set and $\operatorname{diam}\left(\Xi_{l}\right)$ is defined by:

$$
\operatorname{diam}\left(\Xi_{l}\right)=\sup \left\{d\left(\ell^{1}, \ell^{2}\right): \ell^{1}, \ell^{2} \in \Xi_{l}\right\} .
$$

To discuss the existence of fixed point theorems (FPTs) via an MNC for condensing operators, a nice paper was written by Darbo [12]. His results are a generalization of the classical Banach and Schauder FPTs, and he used the theoretical study to present the solutions to differential and integral 
equations. For more published papers that generalized the results of Darbo and more applications, see [13-19].

Darbo's FPT was generalized to coupled and tripled fixed points (FPs) by Rashan [20] and Karakaya et al. [21], respectively. Motivated by these results, here, we generalize Darbo's FPT, and as a consequence, we obtain the existence of QFPs. Here, we use the following symbols:

- Via a norm $\|\cdot\|, Z$ refers to a Banach space (BS),

- At the center $\ell^{1}$ and radius $\epsilon, S\left(\ell^{1}, \epsilon\right)$ is a closed ball,

- The algebraic operations on sets take the form $\varkappa Q$ and $Q+P$, where $P, Q \in Z$ and $\varkappa \in \mathbb{R}$,

- $\bar{Q}$ refers to the closure of a set $Q$,

- $\quad c o(Q)$ and $\overline{c o}(Q)$ stand for the convex hull and closed convex hull of $Q$, respectively,

- $\Theta_{Z}$ symbolizes the collection of all bounded nonempty subsets of a BS Z,

- $\Theta_{Z}^{\prime}$ is a subfamily composed of all relatively compact subsets of $Z$,

- $\quad \Xi$ is a nonempty, closed, bounded, and convex (NCBC) subset of a BS Z.

In this manuscript, the existence of the solution of nonlinear functional integral equations in the Banach space under the technique of a measure of noncompactness is obtained. Furthermore, some quadruple fixed point results that generalize Darbo's fixed point theorem are given. Ultimately, some examples to illustrate our study are included.

\section{Preliminaries}

Now, we recall some of the elementary useful results in our study.

Definition 1. [22] A function $\mho: \Theta_{Z} \rightarrow[0,+\infty)$ is called an $M N C$ in $Z$ if the stipulations below are fulfilled:

(i) The family $\operatorname{ker}(\mho)=\left\{Q \in \Theta_{Z}: \mho(Q)=0\right\} \neq \varnothing$ and $\operatorname{ker}(\mho) \subset \Theta_{Z}^{\prime}$;

(ii) if $Q \subset P$, then $\mho(Q) \leq \mho(P)$;

(iii) $\mho(Q)=\mho(\bar{Q})$;

(iv) $\mho(\operatorname{coQ} Q)=\mho(Q)$;

(v) for all $\pi \in[0,1], \mho(\pi Q+(1-\pi) P)=\pi \mho(Q)+(1-\pi) \mho(P)$;

(vi) if $\lim _{n \rightarrow \infty} \mho\left(Q_{n}\right)=0$, where $Q_{n} \in \Theta_{Z}$ is a sequence of closed sets so that, for $n \in \mathbb{N}, Q_{n+1} \subset Q_{n}$, then $Q_{\infty}=\cap_{n=1}^{\infty} Q_{n} \neq \varnothing$.

Note that $\operatorname{ker}(\mho)$ mentioned in (i) is called a kernel of an MNC $\mho$, and $Q_{\infty}$ described in (vi) is an element of the collection $\operatorname{ker}(\mho)$. Furthermore, we can see $\mho\left(Q_{\infty}\right)=0$ because $\mho\left(Q_{\infty}\right) \subset \mho\left(Q_{n}\right)$ for all $n$. Thus, $Q_{\infty} \in \operatorname{ker}(\mho)$.

Definition 2. [23] Assume that $Q$ and $P$ are two BSs; the mapping $\Im: Q \rightarrow P$ is called compact if for any bounded subset $Y$ in $Q, \Im(Y)$ is relatively compact in $P$.

Theorem 1. [10] Let $\Xi$ be an NCBC subset of a BS Z. If the map $\Im: \Xi \rightarrow \Xi$ is continuous and compact, then it has one FP on $\Xi$.

Theorem 2. [12] Assume that $\Xi$ is an NCBC subset of $a B S Z$ and $\Im: \Xi \rightarrow \Xi$ be a continuous mapping so that $0 \leq \delta<1$ and:

$$
\mho(\Im Y) \leq \delta \mho(Y)
$$

for each non-empty subset $Y$ of $\Xi$. Then, on $\Xi$, $\Im$ has an FP.

The idea of coupled fixed points and mixed-monotone functions in partially ordered metric spaces was first introduced in paper [24]. Under abstract spaces, some main consequences in the same lines have been incorporated (see, for example, [25-29]). 
Definition 3. [24] We say that $\left(\ell^{1}, \ell^{2}\right) \in Z^{2}$ is a coupled FP of the mapping $\Im: Z^{2} \rightarrow Z$, if $\Im\left(\ell^{1}, \ell^{2}\right)=\ell^{1}$ and $\Im\left(\ell^{2}, \ell^{1}\right)=\ell^{2}$.

Lemma 1. [30] Assume that $\mho_{1}, \mho_{1}, \ldots, \mho_{n}$ are an $M N C$ in $B S s Z_{1}, Z_{2}, \ldots, Z_{n}$, respectively. Consider a convex function $K:[0, \infty)^{n} \rightarrow[0, \infty)$ so that $K\left(\ell^{1}, \ell^{2}, \ldots, \ell^{n}\right)=0$ iff $\ell^{i}=0, \forall i=1,2, \ldots, n$. Therefore, an MNC in $Z_{1} \times Z_{2} \times \ldots \times Z_{n}$ is defined as follows:

$$
\mho(Y)=K\left(\mho_{1}\left(Y_{1}\right), \mho_{2}\left(Y_{2}\right), \ldots, \mho_{n}\left(Y_{n}\right)\right),
$$

for a natural projection $Y_{i}$ of $Y$ into $Z_{i}$.

Here, if $Y \neq \varnothing$ is a subset of $Z^{j}$, where $Z$ is a BS, we shall write that $Y_{i}$ refers to the image $\pi_{i}(Y)$ for $i=1,2, \ldots, n$, where $\pi\left(\ell^{1}, \ell^{2}, \ldots, \ell^{j}\right)=\ell^{i},\left(\ell^{1}, \ell^{2}, \ldots, \ell^{j}\right) \in Y$.

The following family of functions was introduced by Roshan [20]:

Definition 4. Suppose that $\Psi$ is a family of all functions $\psi: \mathbb{R}_{+}^{2} \rightarrow \mathbb{R}_{+}$, with usual order relation " $\leq$" on $\mathbb{R}_{+}^{2}=\mathbb{R}_{+} \times \mathbb{R}_{+}$as $\left.\left(\ell^{1}, \ell^{2}\right) \leq(\urcorner^{1}, \tau^{2}\right)$ iff $\ell^{1} \leq \tau^{1}$ and $\ell^{2} \leq \tau^{2}$ satisfying the stipulations below:

- $\quad$ the function $\psi$ is nondecreasing and continuous on $\mathbb{R}_{+}^{2}$;

- for all $\ell>0, \psi(\ell, \ell)<\ell$;

- for all $\left\{\ell^{i}, 7^{i}: i=1,2\right\} \in \mathbb{R}_{+}$,

$$
\frac{1}{2} \psi\left(\ell^{1}, \ell^{2}\right)+\frac{1}{2} \psi\left(\neg^{1}, \neg^{2}\right) \leq \psi\left(\frac{\left.\ell^{1}+\right\urcorner^{1}}{2}, \frac{\left.\ell^{2}+\right\urcorner^{2}}{2}\right) .
$$

Theorem 3. [20] Let $\Xi$ be an NCBC subset of a BS Z and $\mho$ be an arbitrary MNC on Z. Consider $\mho^{*}$ as in Lemma 1 and $\psi \in \Psi$. Assume that $\Im: \Xi^{2} \rightarrow Y^{2}$ is a continuous function satisfying:

$$
\mho^{*}(\Im(Y))=\psi\left(\mho^{*}(Y), \mho^{*}(Y)\right)
$$

for any non-empty subset $Y$ of $\Xi \times \Xi$. Then, $\Im$ has an $F P$.

In 2011, triple fixed points (TFPs) were initiated in [21,31] for self-mappings in partially ordered metric spaces and condensing operators in Banach spaces. Nice results are given by these points. For instance, see [32-35].

Definition 5. [21,31] Assume that $\Im: Z^{3} \rightarrow Z$ is a given mapping. We say that $\left(\ell^{1}, \ell^{2}, \ell^{3}\right) \in Z^{3}$ is a tripled FP of $\Im$, if $\Im\left(\ell^{1}, \ell^{2}, \ell^{3}\right)=\ell^{1}, \Im\left(\ell^{2}, \ell^{3}, \ell^{1}\right)=\ell^{2}$, and $\Im\left(\ell^{3}, \ell^{1}, \ell^{2}\right)=\ell^{3}$.

Karapinar [36] initiated the idea of quadruple fixed points (QFPs) as a generalization of TFPs. He presented some fixed point results on the topic. Following this study, a QFP is upgraded, and some related fixed point consequences were shown in [37-40].

Definition 6. [36] Assume that $\Im: Z^{4} \rightarrow Z$ is a given mapping, we say that $\left(\ell^{1}, \ell^{2}, \ell^{3}, \ell^{4}\right) \in Z^{3}$ is a QFP of $\Im$, if $\Im\left(\ell^{1}, \ell^{2}, \ell^{3}, \ell^{4}\right)=\ell^{1}, \Im\left(\ell^{2}, \ell^{3}, \ell^{4}, \ell^{1}\right)=\ell^{2}, \Im\left(\ell^{3}, \ell^{4}, \ell^{1}, \ell^{2}\right)=\ell^{3}$, and $\Im\left(\ell^{4}, \ell^{1}, \ell^{2}, \ell^{3}\right)=\ell^{4}$. 


\section{Results}

We begin this part with the important examples as a result of Lemma 1 as follows:

Example 1. Suppose that $\mho$ is an MNC on a BS $Z, K: \mathbb{R}_{+}^{4} \rightarrow \mathbb{R}_{+}$is a convex function, and $K\left(\ell^{1}, \ell^{2}, \ell^{3}, \ell^{4}\right)=$ 0 iff $\ell^{j}=0, \forall j=1,2,3,4$. Then:

$$
\mho^{*}(Y)=K\left(\mho\left(Y_{1}\right), \mho\left(Y_{2}\right), \mho\left(Y_{3}\right), \mho\left(Y_{4}\right)\right)
$$

defines an $M N C$ in $Z^{4}=Z \times Z \times Z \times Z$.

Example 2. Assume that $\mho$ is an MNC on a BS Z,K: $\mathbb{R}_{+}^{4} \rightarrow \mathbb{R}_{+}$is a convex function defined by $K\left(\ell^{1}, \ell^{2}, \ell^{3}, \ell^{4}\right)=\sum_{j}^{4} \ell^{j}$. Then:

$$
\mho^{*}(Y)=\mho\left(Y_{1}\right)+\mho\left(Y_{2}\right)+\mho\left(Y_{3}\right)+\mho\left(Y_{4}\right)
$$

defines an MNC in $Z^{4}$.

Example 3. Assume that $\mho$ is an MNC on a BS Z,K: $\mathbb{R}_{+}^{4} \rightarrow \mathbb{R}_{+}$is a convex function defined by $K\left(\ell^{1}, \ell^{2}, \ell^{3}, \ell^{4}\right)=\max \left\{\ell^{1}, \ell^{2}, \ell^{3}, \ell^{4}\right\}$ for all $\left(\ell^{1}, \ell^{2}, \ell^{3}, \ell^{4}\right) \in \mathbb{R}_{+}^{4}$. Then:

$$
\mho^{*}(Y)=\max \left\{\mho\left(Y_{1}\right), \mho\left(Y_{2}\right), \mho\left(Y_{3}\right), \mho\left(Y_{4}\right)\right\}
$$

is an MNC in the space $\mathrm{Z}^{4}$.

The functions below are more general than the functions of Roshan [20].

Definition 7. Assume that " $\leq$ " is an order relation defined on $\mathbb{R}_{+}^{4}$ by:

$$
\left(\ell^{1}, \ell^{2}, \ell^{3}, \ell^{4}\right) \leq\left(\neg^{1}, \neg^{2}, \neg^{3}, \neg^{4}\right) \text { iff } \ell^{1} \leq \neg^{1}, \ell^{2} \leq \neg^{2}, \ell^{3} \leq \neg^{3} \text { and } \ell^{4} \leq \neg^{4}
$$

Assume that $\Re$ is the family of all functions $\varphi: \mathbb{R}_{+}^{4} \rightarrow \mathbb{R}_{+}$, verifying the stipulations below.

$\Re_{1}$. the function $\varphi$ is nondecreasing and continuous on $\mathbb{R}_{+}^{4}$;

$\Re_{2}$. for all $\ell>0, \varphi(\ell, \ell, \ell, \ell)<\ell$;

$\Re_{3}$. for all $\ell^{1}, \ell^{2}, \ell^{3}, \ell^{4} \in \mathbb{R}_{+}, \varphi\left(\ell^{1}, \ell^{2}, \ell^{3}, \ell^{4}\right)=\varphi\left(\ell^{2}, \ell^{3}, \ell^{4}, \ell^{1}\right)=\varphi\left(\ell^{3}, \ell^{4}, \ell^{1}, \ell^{2}\right)=\varphi\left(\ell^{4}, \ell^{1}, \ell^{2}, \ell^{3}\right)$;

$\Re_{4}$. for all $\left\{\ell^{j}, 7^{j}, p^{j}, u^{j}: j=1,2,3,4\right\} \in \mathbb{R}_{+}$,

$$
\begin{aligned}
& \frac{1}{4} \varphi\left(\ell^{1}, \ell^{2}, \ell^{3}, \ell^{4}\right)+\frac{1}{4} \varphi\left(\neg^{1}, \neg^{2}, \neg^{3}, \neg^{4}\right)+\frac{1}{4} \varphi\left(p^{1}, p^{2}, p^{3}, p^{4}\right)+\frac{1}{4} \varphi\left(u^{1}, u^{2}, u^{3}, u^{4}\right) \\
\leq & \varphi\left(\frac{\ell^{1}+\neg^{1}+p^{1}+u^{1}}{4}, \frac{\ell^{2}+\neg^{2}+p^{2}+u^{2}}{4}, \frac{\ell^{3}+\neg^{3}+p^{3}+u^{3}}{4}, \frac{\ell^{4}+\neg^{4}+p^{4}+u^{4}}{4}\right) .
\end{aligned}
$$

For example, all functions below are members of $\Re$ :

- $\varphi\left(\ell^{1}, \ell^{2}, \ell^{3}, \ell^{4}\right)=a_{1} \ell^{1}+a_{2} \ell^{2}+a_{3} \ell^{3}+a_{4} \ell^{4}$, for all $0 \leq a_{1}, a_{2}, a_{3}, a_{4}<1$ so that $\sum_{j=1}^{4} a_{j}<1$,

- $\varphi\left(\ell^{1}, \ell^{2}, \ell^{3}, \ell^{4}\right)=\ln \left(1+\frac{\ell^{1}+\ell^{2}+\ell^{3}+\ell^{4}}{4}\right)$,

- $\varphi\left(\ell^{1}, \ell^{2}, \ell^{3}, \ell^{4}\right)=\frac{1}{5}\left(\ell^{1}+\ell^{2}+\ell^{3}+\ell^{4}\right)$. 
Theorem 4. Assume that $\Xi$ is an NCBC subset of a BS Z and $\mho$ is an arbitrary MNC on Z. Consider $\mho^{*}$ as in Example 1, and $\varphi \in \Re$. Suppose that $\Im: \Xi^{4} \rightarrow \Xi^{4}$ is a continuous function verifying:

$$
\mho^{*}(\Im(Y)) \leq \varphi\left(\mho^{*}(Y), \mho^{*}(Y), \mho^{*}(Y), \mho^{*}(Y)\right),
$$

for any non-empty subset $Y$ of $\Xi^{4}$. Then, $\Im$ has at least one FP in $\Xi^{4}$, and the set of all FPs of $\Im$ is compact.

Proof. Consider $B_{0}=\Xi^{4}$, and define a sequence $B_{n}=\overline{c o} \Im\left(B_{n-1}\right), n \in \mathbb{N}$.

Firstly, we note that:

$$
\begin{aligned}
\mho^{*}\left(B_{n+1}\right) & =\mho^{*}\left(\overline{c o}\left(\Im\left(B_{n}\right)\right)\right) \\
& =\mho^{*}\left(\Im\left(B_{n}\right)\right) \\
& \leq \varphi\left(\mho^{*}\left(B_{n}\right), \mho^{*}\left(B_{n}\right), \mho^{*}\left(B_{n}\right), \mho^{*}\left(B_{n}\right)\right) .
\end{aligned}
$$

Secondly, $B_{1}=\overline{c o}\left(\Im\left(B_{0}\right)\right)=\overline{c o}\left(\Im\left(\Xi^{4}\right)\right) \subset \mathrm{Y}^{4}=B_{0}$; similarly, $B_{2} \subset B_{1}$. Now, if $B_{n} \subset B_{n-1}$, then $\Im B_{n} \subset \Im B_{n-1}$, which leads to:

$$
\Im B_{n} \cup B_{n+1}=\overline{c o}\left(\Im\left(B_{n}\right)\right) \subset \overline{c o}\left(\Im\left(B_{n-1}\right)\right)=B_{n} .
$$

Hence, we conclude that the sequence $\mho^{*}\left(B_{n}\right)$ is a non-increasing of $\mathbb{R}$; thus, there is a constant $\ell \geq 0$ so that $\lim _{n \rightarrow \infty} \mho^{*}\left(B_{n}\right)=\ell$. We shall illustrate that $\ell=0$. Let us suppose that $\ell>0$. Then, it holds by (2), and we get:

$$
\begin{aligned}
\ell & =\lim _{n \rightarrow \infty} \mho^{*}\left(B_{n+1}\right) \\
& \leq \lim _{n \rightarrow \infty} \varphi\left(\mho^{*}\left(B_{n}\right), \mho^{*}\left(B_{n}\right), \mho^{*}\left(B_{n}\right), \mho^{*}\left(B_{n}\right)\right) \\
& \leq \varphi\left(\lim _{n \rightarrow \infty} \mho^{*}\left(B_{n}\right), \lim _{n \rightarrow \infty} \mho^{*}\left(B_{n}\right), \lim _{n \rightarrow \infty} \mho^{*}\left(B_{n}\right), \lim _{n \rightarrow \infty} \mho^{*}\left(B_{n}\right)\right) \\
& =\varphi(\ell, \ell, \ell, \ell)<\ell,
\end{aligned}
$$

which is a contradiction; so, we get $\lim _{n \rightarrow \infty} \mho^{*}\left(B_{n}\right)=0$. Since $B_{n+1} \subset B_{n}$, then by the stipulation (vi) of Definition 1, we obtain that $B_{\infty}=\cap_{n=1}^{\infty} B_{n}$ is an NCC, invariant via $\Im$, and contained in $\operatorname{ker}\left(\mho^{*}\right)$. Thus, Theorem 1 tells us that the mapping $\Im$ has an FP in $B_{\infty}$.

Next, assume that $\nabla$ is the set of all FPs of $\Im$. If $\mho^{*}(\nabla)>0$, then by (1), one can write:

$$
\mho^{*}(\Im(\nabla)) \leq \varphi\left(\mho^{*}(\nabla), \mho^{*}(\nabla), \mho^{*}(\nabla), \mho^{*}(\nabla)\right)<\mho^{*}(\nabla),
$$

which is a contradiction since $\Im(\nabla)=\nabla$. Thus, $\mho^{*}(\nabla)=0$, and this leads to the fact that $\nabla$ is relatively compact.

Finally, consider $\left\{\ell_{n}^{1}\right\} \subset \nabla$ to be a convergent sequence so that $\ell_{n}^{1} \rightarrow \ell^{1}$, and since $B_{0}$ is closed, then we get $\ell^{1} \in B_{0}$. Furthermore, $\Im$ is continuous, so we can write $\ell_{n}^{1}=\Im \ell_{n}^{1} \rightarrow \ell^{1}$ and $\Im \ell^{1}=\ell^{1}$, which leads to $\ell^{1} \in \nabla$. Therefore, $\nabla$ is a compact set.

Remark 1. Theorem 4 is valid if we replace the stipulation (1) by the conditions below: For all $0 \leq a_{1}, a_{2}, a_{3}, a_{4}<1$, so that $\sum_{j=1}^{4} a_{j}<1$,

- $\quad \varphi\left(\mho^{*}(Y), \mho^{*}(Y), \mho^{*}(Y), \mho^{*}(Y)\right)=a_{1} \mho^{*}(Y)+a_{2} \mho^{*}(Y)+a_{3} \mho^{*}(Y)+a_{4} \mho^{*}(Y)$;

- there is a function $\lambda: \mathbb{R}_{+} \rightarrow[0,1)$, so that $\lambda\left(u_{n}\right) \rightarrow 1$ implies $u_{n} \rightarrow 0$ and:

$$
\begin{aligned}
\varphi\left(\mho^{*}(Y), \mho^{*}(Y), \mho^{*}(Y), \mho^{*}(Y)\right)= & a_{1} \lambda\left(\mho^{*}(Y)\right) \mho^{*}(Y)+a_{2} \lambda\left(\mho^{*}(Y)\right) \mho^{*}(Y) \\
& +a_{3} \lambda\left(\mho^{*}(Y)\right) \mho^{*}(Y)+a_{4} \lambda\left(\mho^{*}(Y)\right) \mho^{*}(Y)
\end{aligned}
$$


- there is an upper semi-continuous and non-decreasing function $\varrho: \mathbb{R}_{+} \rightarrow \mathbb{R}_{+}$so that $\varrho(v)<v$ for $v>0$ and:

$$
\varphi\left(\mho^{*}(Y), \mho^{*}(Y), \mho^{*}(Y), \mho^{*}(Y)\right)=a_{1} \varrho\left(\mho^{*}(Y)\right)+a_{2} \varrho\left(\mho^{*}(Y)\right)+a_{3} \varrho\left(\mho^{*}(Y)\right)+a_{4} \varrho\left(\mho^{*}(Y)\right)
$$

Now, the results concerned with the QFP of a mapping $\Im$ become valid for discussion.

Theorem 5. Assume that $\Xi$ is an NCBC subset of a BS Z. Let $\mho$ be an arbitrary MNC on Z. Assume a continuous function $\Im_{i}: Y^{4} \rightarrow \Xi, \forall i=1,2,3,4$ verifying:

$$
\mho\left(\Im_{i}(\Delta)\right) \leq \varphi\left(\mho\left(Y^{1}\right), \mho\left(Y^{2}\right), \mho\left(Y^{3}\right), \kappa\left(Y^{4}\right)\right)
$$

where $\varphi \in \Re$, and for any non-empty subset $Y^{1}, Y^{2}, Y^{3}, Y^{4}$ of $\Xi$ and $\Delta=Y^{1} \times Y^{2} \times Y^{3} \times Y^{4}$. Then, there is an element $\left(\ell^{* 1}, \ell^{* 2}, \ell^{* 3}, \ell^{\prime 4}\right) \in \Xi^{4}$, so that:

$$
\begin{aligned}
& \ell^{* 1}=\Im_{1}\left(\ell^{* 1}, \ell^{* 2}, \ell^{* 3}, \ell^{* 4}\right), \ell^{* 2}=\Im_{2}\left(\ell^{* 1}, \ell^{* 2}, \ell^{* 3}, \ell^{* 4}\right), \\
& \ell^{* 3}=\Im_{3}\left(\ell^{* 1}, \ell^{* 2}, \ell^{* 3}, \ell^{* 4}\right), \ell^{* 4}=\Im_{4}\left(\ell^{* 1}, \ell^{* 2}, \ell^{* 3}, \ell^{* 4}\right) .
\end{aligned}
$$

Proof. Define the operator $\aleph: \Xi^{4} \rightarrow \Xi$ by:

$$
\aleph\left(\ell^{1}, \ell^{2}, \ell^{3}, \ell^{4}\right)=\left(\Im_{1}\left(\ell^{1}, \ell^{2}, \ell^{3}, \ell^{4}\right), \Im_{2}\left(\ell^{1}, \ell^{2}, \ell^{3}, \ell^{4}\right), \Im_{3}\left(\ell^{1}, \ell^{2}, \ell^{3}, \ell^{4}\right), \Im_{4}\left(\ell^{1}, \ell^{2}, \ell^{3}, \ell^{4}\right)\right) .
$$

It follows from Example 2 that:

$$
\mho^{*}(Y)=\mho\left(Y_{1}\right)+\mho\left(Y_{2}\right)+\mho\left(Y_{3}\right)+\mho\left(Y_{4}\right),
$$

is an MNC in $Z^{4}$. Clearly, on $\Xi^{4}$, the operator $\aleph$ is continuous. Only here, we want to show that $\aleph$ has a fixed point by showing that $\aleph$ verifies the stipulation (1) of Theorem 4. Consider $Y \subset \Xi^{4}$; we get:

$$
\begin{aligned}
\mho^{*}(\aleph(Y)) \leq & \mho^{*}\left(\Im_{1}(\Delta) \times \Im_{2}(\Delta) \times \Im_{3}(\Delta) \times \Im_{4}(\Delta)\right) \\
= & \mho\left(\Im_{1}(\Delta)\right)+\mho\left(\Im_{2}(\Delta)\right)+\mho\left(\Im_{3}(\Delta)\right)+\mho\left(\Im_{4}(\Delta)\right) \\
\leq & \varphi\left(\mho\left(Y^{1}\right), \mho\left(Y^{2}\right), \mho\left(Y^{3}\right), \mho\left(Y^{4}\right)\right)+\varphi\left(\mho\left(Y^{1}\right), \mho\left(Y^{2}\right), \mho\left(Y^{3}\right), \mho\left(Y^{4}\right)\right) \\
& +\varphi\left(\mho\left(Y^{1}\right), \mho\left(Y^{2}\right), \mho\left(Y^{3}\right), \mho\left(Y^{4}\right)\right)+\varphi\left(\mho\left(Y^{1}\right), \mho\left(Y^{2}\right), \mho\left(Y^{3}\right), \mho\left(Y^{4}\right)\right) \\
\leq & 4 \varphi\left(\frac{\mho\left(Y^{1}\right)+\mho\left(Y^{2}\right)+\mho\left(Y^{3}\right)+\mho\left(Y^{4}\right)}{\frac{\mho\left(Y^{1}\right)+\mho\left(Y^{2}\right)+\mho\left(Y^{3}\right)+\mho\left(Y^{4}\right)}{4}, \frac{\mho\left(Y^{1}\right)+\mho\left(Y^{2}\right)+\mho\left(Y^{3}\right)+\mho\left(Y^{4}\right)}{4},}\right) \\
= & 4 \varphi\left(\frac{\mho^{*}(Y)+\mho\left(Y^{3}\right)+\mho\left(Y^{4}\right)}{4}, \frac{\mho^{*}(Y)}{4}, \frac{\mho^{*}(Y)}{4}, \frac{\mho^{*}(Y)}{4}\right) .
\end{aligned}
$$

Put $\mho_{1}^{*}=\frac{1}{4} \mho^{*}$ in (6); we have:

$$
\mho_{1}^{*}(\aleph(Y)) \leq \varphi\left(\mho_{1}^{*}(Y), \mho_{1}^{*}(Y), \mho_{1}^{*}(Y), \mho_{1}^{*}(Y)\right) .
$$

Thus, by Theorem 4, $\aleph$ has an FP. 


\section{Remark 2.}

(a) Clearly, the stipulation:

$$
\mho\left(\Im_{i}(Y)\right) \leq \varphi\left(\mho\left(Y^{1}\right), \mho\left(Y^{2}\right), \mho\left(Y^{3}\right), \mho\left(Y^{4}\right)\right),
$$

for a non-empty subset Yof $\Xi^{4}$, is equivalent to the stipulation (4) because:

$$
\mho\left(\Im_{i}(Y)\right) \leq \mho\left(\Im_{i}(\Delta)\right),
$$

where $\Delta=Y^{1} \times Y^{2} \times Y^{3} \times Y^{4}$.

(b) Theorem 5 is still valid if we take the function below:

$$
\varphi\left(\mho\left(Y^{1}\right), \mho\left(Y^{2}\right), \mho\left(Y^{3}\right), \mho\left(Y^{4}\right)\right) \leq \lambda \max \left\{\mho\left(Y^{i}\right): i=1,2,3,4\right\},
$$

where $\lambda \in(0,1)$.

Corollary 1. Let $\Xi$ be an NCBC subset of a BS Z and $\mho$ be an arbitrary MNC on Z. Assume that $\Im: \Xi^{4} \rightarrow \Xi$ is a continuous function verifying:

$$
\mho(\Im(\Delta)) \leq \varphi\left(\mho\left(Y^{1}\right), \mho\left(Y^{2}\right), \mho\left(Y^{3}\right), \mho\left(Y^{4}\right)\right)
$$

where $\varphi \in \Re$ and for any non-empty subset $Y^{1}, Y^{2}, Y^{3}, Y^{4}$ of $\Xi$ and $\Delta$ defined in Theorem 5 . Then, $\Im$ has at least a QFP, that is there is $\left(\ell^{* 1}, r^{* 2}, \ell^{* 3}, \ell^{* 4}\right) \in \Xi^{4}$ so that:

$$
\begin{aligned}
& \ell^{* 1}=\Im\left(\ell^{* 1}, \ell^{* 2}, \ell^{* 3}, \ell^{* 4}\right), \ell^{* 2}=\Im\left(\ell^{* 2}, \ell^{* 3}, \ell^{* 4}, \ell^{* 1}\right), \\
& \ell^{* 3}=\Im\left(\ell^{* 3}, \ell^{* 4}, \ell^{* 1}, \ell^{* 2},\right), \ell^{* 4}=\Im_{4}\left(\ell^{* 4}, \ell^{* 1}, \ell^{* 2}, \ell^{* 3}\right) .
\end{aligned}
$$

Proof. From Theorem 5, the proof follows immediately, by setting $\Im_{i}=\Im$, for all $i=1,2,3,4$ and:

$$
\aleph\left(\ell^{1}, \ell^{2}, \ell^{3}, \ell^{4}\right)=\left(\Im\left(\ell^{1}, \ell^{2}, \ell^{3}, \ell^{4}\right), \Im\left(\ell^{2}, \ell^{3}, \ell^{4}, \ell^{1}\right), \Im\left(\ell^{3}, \ell^{4}, \ell^{1}, \ell^{2},\right), \Im\left(\ell^{4}, \ell^{1}, \ell^{2}, \ell^{3}\right)\right) .
$$

Corollary 2. Let $\Xi$ be an NCBC subset of a BS Z and $\mho$ be an arbitrary MNC on Z. Moreover, assume that $\Im: \Xi^{4} \rightarrow \Xi$ is a continuous function so that there are constants $0 \leq a_{1}, a_{2}, a_{3}, a_{4}<1$ with $\sum_{j=1}^{4} a_{j}<1$,

$$
\mho(\Im(\Delta)) \leq a_{1} \mho\left(Y^{1}\right)+a_{2} \mho\left(Y^{2}\right)+a_{3} \mho\left(Y^{3}\right)+a_{4} \mho\left(Y^{4}\right),
$$

for any non-empty subset $Y^{1}, Y^{2}, Y^{3}, Y^{4}$ of $Y$. Then, $\Im$ has at least a QFP.

Proof. Putting $\Im_{i}=\Im$, for all $i=1,2,3,4$ and:

$$
\varphi\left(\ell^{1}, \ell^{2}, \ell^{3}, \ell^{4}\right)=a_{1} \ell^{1}+a_{2} \ell^{2}+a_{3} \ell^{3}+a_{4} \ell^{4},
$$

in Theorem 5, we get the proof. 
Theorem 6. Assume that $\Xi$ is an NCBC subset of a BS Z and $\mho$ be an arbitrary MNC on Z. Let $\Im_{i}: \Xi^{4} \rightarrow \Xi$, for $i=1,2,3,4$ be a continuous function justifying:

$$
\mho\left(\Im_{i}(\Delta)\right) \leq \varphi\left(\begin{array}{c}
\max \left\{\mho\left(Y^{1}\right), \mho\left(Y^{2}\right), \mho\left(Y^{3}\right), \mho\left(Y^{4}\right)\right\}, \\
\max \left\{\mho\left(Y^{1}\right), \mho\left(Y^{2}\right), \mho\left(Y^{3}\right), \mho\left(Y^{4}\right)\right\}, \\
\max \left\{\mho\left(Y^{1}\right), \mho\left(Y^{2}\right), \mho\left(Y^{3}\right), \mho\left(Y^{4}\right)\right\}, \\
\max \left\{\mho\left(Y^{1}\right), \mho\left(Y^{2}\right), \mho\left(Y^{3}\right), \mho\left(Y^{4}\right)\right\}
\end{array}\right)
$$

where $\varphi \in \Re$, for any non-empty subset $Y^{1}, Y^{2}, Y^{3}, Y^{4}$ of $Y$ and $\Delta$ defined in Theorem 5. Then, there is an element $\left(\ell^{* 1}, \ell^{* 2}, \ell^{* 3}, \ell^{*} 4\right) \in \Xi^{4}$ so that:

$$
\begin{aligned}
& \ell^{* 1}=\Im_{1}\left(\ell^{* 1}, \ell^{* 2}, \ell^{* 3}, \ell^{* 4}\right), \ell^{* 2}=\Im_{2}\left(\ell^{* 1}, \ell^{* 2}, \ell^{* 3}, \ell^{* 4}\right), \\
& \ell^{* 3}=\Im_{3}\left(\ell^{* 1}, \ell^{* 2}, \ell^{* 3}, \ell^{* 4}\right), \ell^{* 4}=\Im_{4}\left(\ell^{* 1}, \ell^{* 2}, \ell^{* 3}, \ell^{* 4}\right) .
\end{aligned}
$$

Proof. Let $\aleph: \Xi^{4} \rightarrow \Xi$ be an operator defined by (5). Then, by Example 3, we have that:

$$
\mho^{*}(Y)=\max \left\{\mho\left(Y^{1}\right), \mho\left(Y^{2}\right), \mho\left(Y^{3}\right), \mho\left(Y^{4}\right)\right\}
$$

defines an MNC in $Z^{4}$. To finish the proof, we shall illustrate that $\aleph$ has an FP by proving the stipulations of Theorem 4. Let $Y \subset \Xi^{4}$; we have:

$$
\begin{aligned}
\mho^{*}(\aleph(Y)) & \leq \mho^{*}\left(\Im_{1}(\Delta) \times \Im_{2}(\Delta) \times \Im_{3}(\Delta) \times \Im_{4}(\Delta)\right) \\
& =\max \left\{\mho\left(\Im_{1}(\Delta)\right), \mho\left(\Im_{2}(\Delta)\right), \mho\left(\Im_{3}(\Delta)\right), \mho\left(\Im_{4}(\Delta)\right)\right\} \\
& \leq \varphi\left(\begin{array}{c}
\max \left\{\mho\left(Y^{1}\right), \mho\left(Y^{2}\right), \mho\left(Y^{3}\right), \mho\left(Y^{4}\right)\right\}, \max \left\{\mho\left(Y^{1}\right), \mho\left(Y^{2}\right), \mho\left(Y^{3}\right), \mho\left(Y^{4}\right)\right\}, \\
\max \left\{\mho\left(Y^{1}\right), \mho\left(Y^{2}\right), \mho\left(Y^{3}\right), \mho\left(Y^{4}\right)\right\}, \max \left\{\mho\left(Y^{1}\right), \mho\left(Y^{2}\right), \mho\left(Y^{3}\right), \mho\left(Y^{4}\right)\right\}
\end{array}\right) \\
& =\varphi\left(\mho^{*}(Y), \mho^{*}(Y), \mho \mho^{*}(Y), \mho^{*}(Y)\right) .
\end{aligned}
$$

Therefore, by Theorem $4, \aleph$ has an FP.

If we take $\Im_{i}=\Im$, for all $i=1,2,3,4$ and:

$$
\aleph\left(\ell^{1}, \ell^{2}, \ell^{3}, \ell^{4}\right)=\left(\Im\left(\ell^{1}, \ell^{2}, \ell^{3}, \ell^{4}\right), \Im\left(\ell^{2}, \ell^{3}, \ell^{4}, \ell^{1}\right), \Im\left(\ell^{3}, \ell^{4}, \ell^{1}, \ell^{2},\right), \Im_{4}\left(\ell^{4}, \ell^{1}, \ell^{2}, \ell^{3}\right)\right),
$$

in Theorem 6, the consequence below holds.

Corollary 3. Let $\Xi$ be an NCBC subset of a BS Z and $\mho$ be an arbitrary MNC on Z. Let $\Im: \Xi^{4} \rightarrow \Xi$, for $i=1,2,3,4$ be a continuous function justifying:

$$
\mho(\Im(\Delta)) \leq \varphi\left(\begin{array}{c}
\max \left\{\mho\left(Y^{1}\right), \mho\left(Y^{2}\right), \mho\left(Y^{3}\right), \mho\left(Y^{4}\right)\right\}, \\
\max \left\{\mho\left(Y^{1}\right), \mho\left(Y^{2}\right), \mho\left(Y^{3}\right), \mho\left(Y^{4}\right)\right\}, \\
\max \left\{\mho\left(Y^{1}\right), \mho\left(Y^{2}\right), \mho\left(Y^{3}\right), \mho\left(Y^{4}\right)\right\} \\
\max \left\{\mho\left(Y^{1}\right), \mho\left(Y^{2}\right), \mho\left(Y^{3}\right), \mho\left(Y^{4}\right)\right\}
\end{array}\right),
$$

where $\varphi \in \Re$, and for any non-empty subset $Y^{1}, Y^{2}, Y^{3}, Y^{4}$ of $Y$, then $\Im$ has at least a QFP. 


\section{Supportive Application}

We shall dedicate this part to using the results of Corollary 1 to study the existence of solutions to a system of FIEsin the form of:

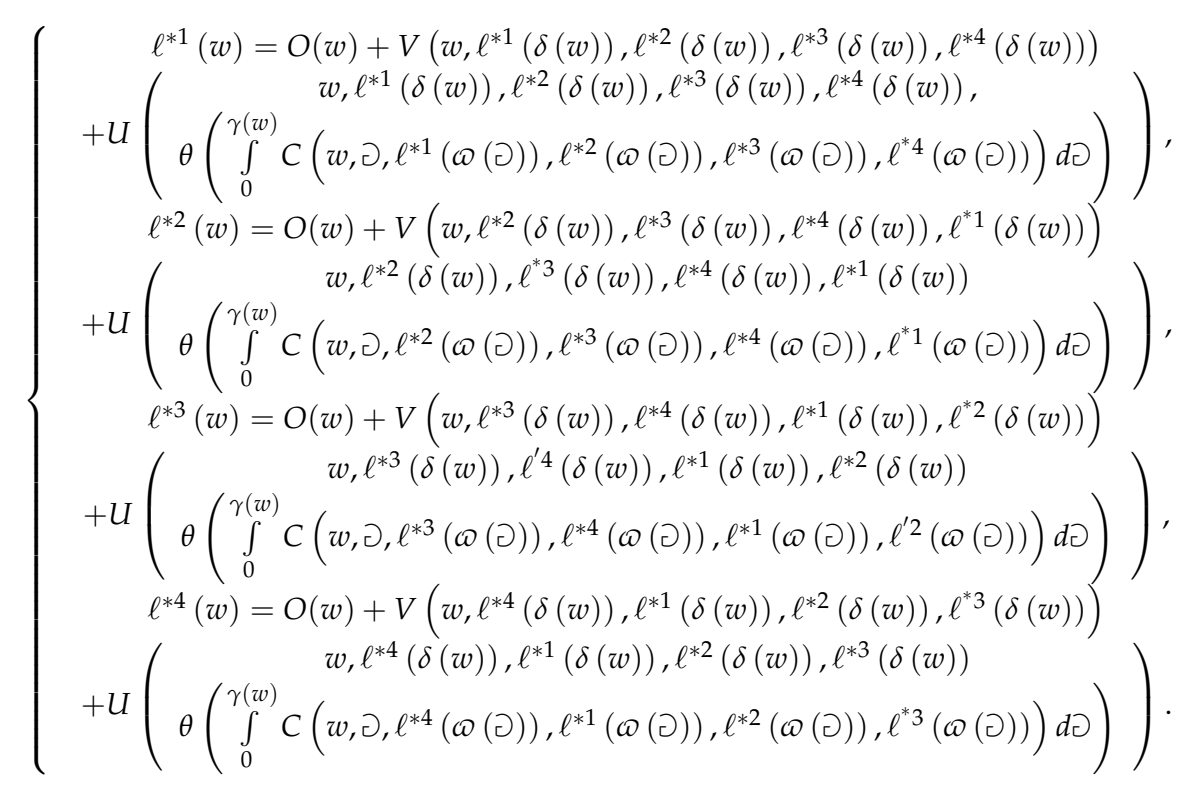

Before discussing the above system, we will introduce some important elementary results.

The modulus of continuity of $\ell^{1} \in Q$ on the closed interval $[0, A]$ is denoted by $\tau^{A}\left(\ell^{1}, \epsilon\right)$ and defined as:

$$
\tau^{A}\left(\ell^{1}, \epsilon\right)=\sup \left\{\left|\ell^{1}(w)-\ell^{2}(\partial)\right|: w, \partial \in[0, A],|w-\partial| \leq \epsilon\right\},
$$

for each $\ell^{1} \in Q$ and $\epsilon>0$, so we can write $\tau^{A}(Q, \epsilon)=\sup \left\{\tau^{A}\left(\ell^{1}, \epsilon\right): \ell^{1} \in Q\right\}$, $\tau_{0}^{A}(Q)=\lim _{\epsilon \rightarrow \infty} \tau^{A}(Q, \epsilon)$, and $\tau_{0}(Q)=\lim _{A \rightarrow \infty} \tau_{0}^{A}(Q)$.

Consider $\chi\left(\mathbb{R}_{+}\right)$to a BS, which consists of all bounded and continuous real-valued functions on $\mathbb{R}_{+}$, and endowed with $\left\|\ell^{1}\right\|_{\infty}=\sup \left\{\left|\ell^{1}(w)\right|: w \geq 0\right\}$. Define an MNC [20-22] on $\Theta_{\chi\left(\mathbb{R}_{+}\right)}$by:

$$
\mho(Q)=\tau_{0}(Q)+\lim \sup _{w \rightarrow \infty} \operatorname{diam} Q(w),
$$

where, for a non-negative fixed $w$,

$$
\operatorname{diam} Q(w)=\sup \left\{\left|\ell^{1}(w)-\ell^{2}(w)\right|: \ell^{1}, \ell^{2} \in Q\right\}
$$

and $Q(w)=\left\{\ell^{1}(w): \ell^{1} \in Q\right\}$.

Now, we return to define the formulas and functions in our system as follows:

$\left(\nabla_{1}\right)$ a function $O: \mathbb{R}_{+} \rightarrow \mathbb{R}$ is bounded continuous with $\xi_{1}=\sup \left\{|O(w)|: w \in \mathbb{R}_{+}\right\} ;$

$\left(\bigcirc_{2}\right) \quad \delta, \omega, \gamma: \mathbb{R}_{+} \rightarrow \mathbb{R}_{+}$are continuous functions and $\delta(w) \rightarrow \infty$ as $w \rightarrow \infty ;$

$\left(Q_{3}\right)$ the functions $V: \mathbb{R}_{+} \times \mathbb{R}^{4} \rightarrow \mathbb{R}$ and $U: \mathbb{R}_{+} \times \mathbb{R}^{5} \rightarrow \mathbb{R}$ are continuous, and for a nondecreasing continuous function $\sigma: \mathbb{R} \rightarrow \mathbb{R}$ with $\sigma(0)=0$ and $\varphi \in \Re$, we have:

$$
\begin{aligned}
& \left.\left.\left.\left.\mid V\left(w, \ell^{* 1}, \ell^{* 2}, \ell^{* 3}, \ell^{* 4}\right)-V(w,\urcorner^{* 1},\right\urcorner^{* 2},\right\urcorner^{* 3},\right\urcorner^{* 4}\right) \mid \\
\leq & \left.\left.\left.\left.\frac{1}{2} \varphi\left(\mid \ell^{* 1}-\right\urcorner^{* 1}|,| \ell^{* 2}-\right\urcorner^{* 2}|,| \ell^{* 3}-\right\urcorner^{* 3}|,| \ell^{* 4}-\right\urcorner^{* 4} \mid\right),
\end{aligned}
$$


and:

$$
\begin{aligned}
& \left.\left.\left.\left.\left.\mid U\left(w, \ell^{* 1}, \ell^{* 2}, \ell^{* 3}, \ell^{* 4}, \ell^{* 5}\right)-U(w,\urcorner^{* 1},\right\urcorner^{* 2},\right\urcorner^{* 3},\right\urcorner^{* 4},\right\urcorner^{* 5}\right) \mid \\
\leq & \left.\left.\frac{1}{2} \varphi\left(\left|\ell^{* 1}-\neg^{* 1}\right|, \mid \ell^{* 2}-\right\urcorner^{* 2}|,| \ell^{* 3}-\right\urcorner^{* 3}|,| \ell^{* 4}-\neg^{* 4} \mid\right)+\sigma\left(\left|\ell^{* 5}-\neg^{* 5}\right|\right),
\end{aligned}
$$

for all $\ell^{*} j, 7^{*} i \in \mathbb{R}$ for $j=1,2,3,4,5$ and for each $w \geq 0$;

$\left(Q_{4}\right)$ the function $\theta: \mathbb{R}_{+} \rightarrow \mathbb{R}$ is continuous with $\theta(0)=0$, and there are $\vartheta, \zeta>0$, so that

$$
\left|\theta\left(w_{1}\right)-\theta\left(w_{2}\right)\right| \leq \vartheta\left|w_{1}-w_{2}\right|^{\zeta}
$$

for each $w_{1}, w_{2} \in \mathbb{R}_{+}$;

$\left(\bigcirc_{5}\right) \quad$ the functions $w \mapsto|U(w, 0,0,0,0,0)|$ and $w \mapsto|V(w, 0,0,0,0)|$ are bounded on $\mathbb{R}_{+}$, that is:

$$
\begin{aligned}
& \xi_{2}=\sup \left\{|U(w, 0,0,0,0,0)|: w \in \mathbb{R}_{+}\right\}<\infty, \\
& \xi_{3}=\sup \left\{|V(w, 0,0,0,0)|: w \in \mathbb{R}_{+}\right\}<\infty ;
\end{aligned}
$$

the function $C: \mathbb{R}_{+} \times \mathbb{R}_{+} \times \mathbb{R}^{4} \rightarrow \mathbb{R}$ is continuous, and there is a positive $d_{0}>0$ so that:

$$
\xi_{1}+\varphi\left(d_{0}, d_{0}, d_{0}, d_{0}\right)+\xi_{2}+\xi_{3}+\sigma\left(\vartheta \xi_{4}\right)<d_{0}
$$

where:

$$
\xi_{4}=\sup \left\{\left|\int_{0}^{\gamma(w)} C\left(w, \partial, \ell^{* 1}(\omega(\partial)), \ell^{* 2}(\omega(\partial)), \ell^{* 3}(\omega(\partial)), \ell^{* 4}(\omega(\partial))\right) d \circlearrowright\right|^{\zeta}\right\},
$$

for all $w \in \mathbb{R}_{+}, \ell^{* 1}, \ell^{* 2}, \ell^{* 3}, \ell^{* 4} \in \chi\left(\mathbb{R}_{+}\right)$and:

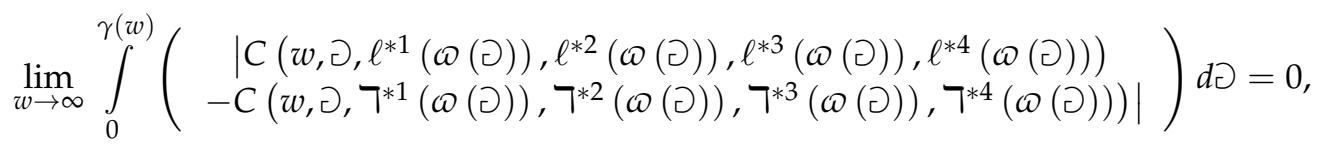

uniformly with respect to $\left.\left.\left.\rceil^{* 1},\right\rceil^{* 2},\right\rceil^{* 3},\right\rceil^{* 4}, \ell^{* 1}, \ell^{* 2}, \ell^{* 3}, \ell^{* 4} \in \chi\left(\mathbb{R}_{+}\right)$.

According to the above stipulations, we present our theorem of this part as follows:

Theorem 7. Via assumptions $\left(\nabla_{1}\right)-\left(\bigcirc_{6}\right)$, System (8) has at least one solution in $\chi^{4}\left(\mathbb{R}_{+}\right)=\chi\left(\mathbb{R}_{+}\right) \times$ $\chi\left(\mathbb{R}_{+}\right) \times \chi\left(\mathbb{R}_{+}\right) \times \chi\left(\mathbb{R}_{+}\right)$.

Proof. Define the operator $\aleph: \chi^{4}\left(\mathbb{R}_{+}\right) \rightarrow \chi\left(\mathbb{R}_{+}\right)$by:

$$
\begin{aligned}
& \aleph\left(\ell^{* 1}, \ell^{* 2}, \ell^{* 3}, \ell^{* 4}\right) \\
& =O(w)+V\left(w, \ell^{* 1}(\delta(w)), \ell^{* 2}(\delta(w)), \ell^{* 3}(\delta(w)), \ell^{* 4}(\delta(w))\right) \\
& +U\left(\theta\left(\int_{0}^{\gamma(w)} C\left(w, \circlearrowright, \ell^{* 1}(\omega(\circlearrowright)), \ell^{* 2}(\mathcal{\omega}(\circlearrowright)), \ell^{* 3}(\omega(\circlearrowright)), \ell^{* 4}(\mathcal{\omega}(\circlearrowright))\right) d \circlearrowright\right)\right),
\end{aligned}
$$


and the space $\chi^{4}\left(\mathbb{R}_{+}\right)$is endowed with the norm below:

$$
\left\|\left(\ell^{* 1}, \ell^{* 2}, \ell^{* 3}, \ell^{* 4}\right)\right\|_{\chi^{4}\left(\mathbb{R}_{+}\right)}=\left\|\ell^{* 1}\right\|_{\infty}+\left\|\ell^{* 2}\right\|_{\infty}+\left\|\ell^{* 3}\right\|_{\infty}+\left\|\ell^{* 4}\right\|_{\infty} .
$$

It is clear that finding a QFP of $\aleph(12)$ is equivalent to solving the system (8), and $\aleph\left(\ell^{* 1}, \ell^{* 2}, \ell^{* 3}, \ell^{* 4}\right)$ is a continuous function for any $\left(\ell^{* 1}, \ell^{* 2}, \ell^{* 3}, \ell^{* 4}\right) \in \chi^{4}\left(\mathbb{R}_{+}\right)$. To finish the proof, we need to fulfill the stipulations of Corollary 1 . Firstly, we show that $\aleph$ is well defined. Therefore, by the triangle inequality and Assumptions $\left(\nabla_{1}\right)-\left(\nabla_{6}\right)$, we have:

$$
\begin{aligned}
& \left|\aleph\left(\ell^{* 1}, \ell^{* 2}, \ell^{* 3}, \ell^{* 4}\right)(w)\right| \\
& \leq|O(w)|+\left|V\left(w, \ell^{* 1}(\delta(w)), \ell^{* 2}(\delta(w)), \ell^{* 3}(\delta(w)), \ell^{* 4}(\delta(w))\right)-V(w, 0,0,0,0)\right| \\
& +|V(w, 0,0,0,0)|+|U(w, 0,0,0,0,0)| \\
& +\left|U\left(\begin{array}{c}
w, \ell^{* 1}(\delta(w)), \ell^{* 2}(\delta(w)), \ell^{* 3}(\delta(w)), \ell^{* 4}(\delta(w)), \\
\theta\left(\int_{0}^{(w)} C\left(w, \partial, \ell^{* 1}(\varpi(\circlearrowright)), \ell^{* 2}(\omega(\circlearrowright)), \ell^{* 3}(\varpi(\circlearrowright)), \ell^{* 4}(\varpi(\circlearrowright))\right) d \circlearrowright\right)
\end{array}\right)-U(w, 0,0,0,0,0)\right| \\
& \leq \xi_{1}+\frac{1}{2} \varphi\left(\left|\ell^{* 1}(\delta(w))\right|,\left|\ell^{* 2}(\delta(w))\right|,\left|\ell^{* 3}(\delta(w))\right|,\left|\ell^{* 4}(\delta(w))\right|\right)+\xi_{3}+\xi_{2} \\
& +\frac{1}{2} \varphi\left(\left|\ell^{* 1}(\delta(w))\right|,\left|\ell^{* 2}(\delta(w))\right|,\left|\ell^{* 3}(\delta(w))\right|,\left|\ell^{* 4}(\delta(w))\right|\right) \\
& +\sigma\left(\left|\theta\left(\int_{0}^{\gamma(w)} C\left(w, \circlearrowright, \ell^{* 1}(\omega(\partial)), \ell^{* 2}(\omega(\circlearrowright)), \ell^{* 3}(\omega(\circlearrowright)), \ell^{* 4}(\omega(\circlearrowright))\right) d \circlearrowright\right)-\theta(0)\right|\right) \\
& \leq \xi_{1}+\varphi\left(\left|\ell^{* 1}(\delta(w))\right|,\left|\ell^{* 2}(\delta(w))\right|,\left|\ell^{* 3}(\delta(w))\right|,\left|\ell^{* 4}(\delta(w))\right|\right)+\xi_{3}+\xi_{2} \\
& +\sigma\left(\vartheta\left|\int_{0}^{\gamma(w)} C\left(w, \partial, \ell^{* 1}(\omega(\circlearrowright)), \ell^{* 2}(\omega(\partial)), \ell^{* 3}(\omega(\partial)), \ell^{* 4}(\omega(\partial))\right) d \circlearrowright\right|^{\zeta}\right) \\
& \leq \xi_{1}+\xi_{2}+\xi_{3}+\varphi\left(\left\|\ell^{* 1}\right\|_{\infty},\left\|\ell^{* 2}\right\|_{\infty},\left\|\ell^{* 3}\right\|_{\infty}\left\|\ell^{* 4}\right\|_{\infty}\right)+\sigma\left(\vartheta \xi_{4}\right) \leq d_{0} .
\end{aligned}
$$

Thus, $\aleph$ is well defined, and we can write $\aleph\left(\left(\bar{O}_{d_{0}}\right)^{4}\right) \subset \bar{O}_{d_{0}}$, where $\left(\bar{O}_{d_{0}}\right)^{4}=\bar{O}_{d_{0}} \times \bar{O}_{d_{0}} \times$ $\bar{O}_{d_{0}} \times \bar{O}_{d_{0}}$.

Secondly, we show that $\aleph:\left(\bar{O}_{d_{0}}\right)^{4} \rightarrow \bar{O}_{d_{0}}$ is continuous. Let $\left(\ell^{* 1}, \ell^{* 2}, \ell^{* 3}, \ell^{* 4}\right)$, (\urcorner$\left.^{* 1}, \boldsymbol{\top}^{* 2}, \boldsymbol{\top}^{* 3}, \boldsymbol{\top}^{* 4}\right) \in\left(\bar{O}_{d_{0}}\right)^{4}$, and $\epsilon>0$ arbitrary. Suppose that:

$$
\left.\|\left(\ell^{* 1}, \ell^{* 2}, \ell^{* 3}, \ell^{* 4}\right)-(\urcorner^{* 1}, \neg^{* 2}, \neg^{* 3}, \neg^{* 4}\right) \|_{\left(\bar{O}_{d_{0}}\right)^{4}}<\frac{\epsilon}{2}
$$

then, one can write:

$$
\begin{aligned}
& \left.\left.\left.\left.\mid \aleph\left(\ell^{* 1}, \ell^{* 2}, \ell^{* 3}, \ell^{* 4}\right)(w)-\aleph(\urcorner^{* 1},\right\rceil^{* 2},\right\rceil^{* 3},\right\rceil^{* 4}\right)(w) \mid \\
& \leq \mid V\left(w, \ell^{* 1}(\delta(w)), \ell^{* 2}(\delta(w)), \ell^{* 3}(\delta(w)), \ell^{* 4}(\delta(w))\right) \\
& \left.-V(w,\rceil^{* 1}(\delta(w)), \boldsymbol{T}^{* 2}(\delta(w)), \boldsymbol{T}^{* 3}(\delta(w)), \boldsymbol{T}^{* 4}(\delta(w))\right) \mid \\
& +\mid U\left(\begin{array}{c}
w, \ell^{* 1}(\delta(w)), \ell^{* 2}(\delta(w)), \ell^{* 3}(\delta(w)), \ell^{* 4}(\delta(w)), \\
\theta\left(\int_{0}^{(w)} C\left(w, \partial, \ell^{* 1}(\omega(\circlearrowright)), \ell^{* 2}(\omega(\circlearrowright)), \ell^{* 3}(\omega(\circlearrowright)), \ell^{* 4}(\omega(\circlearrowright))\right) d \circlearrowright\right)
\end{array}\right)
\end{aligned}
$$




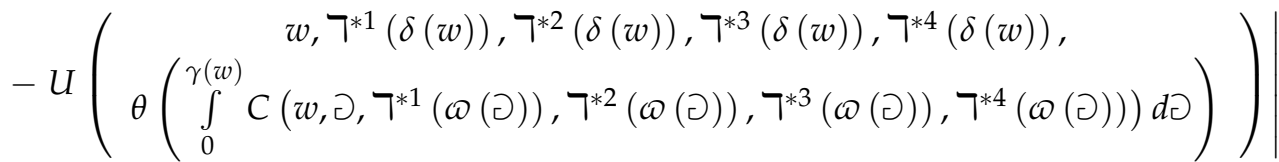

$$
\begin{aligned}
& \leq \varphi\left(\begin{array}{c}
\left|\ell^{* 1}(\delta(w))-\neg^{* 1}(\delta(w))\right|,\left|\ell^{* 2}(\delta(w))-\boldsymbol{T}^{* 2}(\delta(w))\right|, \\
\left|\ell^{* 3}(\delta(w))-\boldsymbol{T}^{* 3}(\delta(w))\right|,\left|\ell^{* 4}(\delta(w))-\boldsymbol{T}^{* 4}(\delta(w))\right|
\end{array}\right) \\
& +\sigma\left(\mid \theta\left(\int_{0}^{\gamma(w)} C\left(w, \partial, \ell^{* 1}(\omega(\partial)), \ell^{* 2}(\omega(\partial)), \ell^{* 3}(\omega(\partial)), \ell^{* 4}(\omega(\partial))\right) d \partial\right)\right. \\
& \left.\left.-\theta\left(\int_{0}^{\gamma(w)} C\left(w, \partial, \boldsymbol{\top}^{* 1}(\omega(\partial)), \boldsymbol{\top}^{* 2}(\omega(\partial)), \boldsymbol{\top}^{* 3}(\omega(\partial)), \boldsymbol{\top}^{* 4}(\omega(\partial))\right) d \partial\right)\right)\right) \\
& \leq \varphi\left(\left\|\ell^{* 1}-\neg^{* 1}\right\|,\left\|\ell^{* 2}-\neg^{* 2}\right\|,\left\|\ell^{* 3}-\neg^{* 3}\right\|,\left\|r^{\prime}-\neg^{* 4}\right\|\right) \\
& +\sigma\left(\vartheta \mid \int_{0}^{\gamma(w)} C\left(w, \partial, \ell^{* 1}(\omega(\partial)), \ell^{* 2}(\omega(\partial)), \ell^{* 3}(\omega(\partial)), \ell^{* 4}(\omega(\partial))\right) d \partial\right. \\
& \left.-\left.\int_{0}^{\gamma(w)} C\left(w, \partial, T^{* 1}(\omega(\partial)), 7^{* 2}(\omega(\partial)), 7^{* 3}(\omega(\partial)), 7^{* 4}(\omega(\partial))\right) d \partial\right|^{\zeta}\right) .
\end{aligned}
$$

Equation (11) leads to there being $A>0$, so that if $w>A$, then:

$$
\begin{aligned}
& \sigma\left(\vartheta \mid \int_{0}^{\gamma(w)} C\left(w, \partial, \ell^{* 1}(\omega(\partial)), \ell^{* 2}(\omega(\partial)), \ell^{* 3}(\omega(\partial)), \ell^{* 4}(\omega(\partial))\right) d \partial\right. \\
& \left.\left.\quad-\theta\left(\int_{0}^{\gamma(w)} C(w, \partial,\rceil^{* 1}(\omega(\partial)), \neg^{* 2}(\omega(\partial)), \nearrow^{* 3}(\omega(\partial)), \nearrow^{* 4}(\omega(\partial))\right) d \partial\right)\left.\right|^{\zeta}\right) \\
& \leq \frac{\epsilon}{2} .
\end{aligned}
$$

for each $\ell^{* 1}, \ell^{* 2}, \ell^{* 3}, \ell^{* 4}, 7^{* 1}, 7^{* 2}, 7^{* 3}, 7^{* 4} \in \bar{O}_{d_{0}}$. Here, there are two cases:

Case 1. If $w>A$, then by (13) and (15), we get:

$$
\begin{aligned}
& \left|\aleph\left(\ell^{* 1}, \ell^{* 2}, \ell^{* 3}, \ell^{* 4}\right)(w)-\aleph\left(\neg^{* 1}, 7^{* 2}, 7^{* 3}, 7^{* 4}\right)(w)\right| \\
\leq & \varphi\left(\frac{\epsilon}{2}, \frac{\epsilon}{2}, \frac{\epsilon}{2}, \frac{\epsilon}{2}\right)+\frac{\epsilon}{2}<\frac{\epsilon}{2}+\frac{\epsilon}{2}=\epsilon .
\end{aligned}
$$

Case 2. If $w \in[0, A]$, then similarly, we can write:

$$
\begin{aligned}
& \left|\aleph\left(\ell^{* 1}, \ell^{* 2}, \ell^{* 3}, \ell^{* 4}\right)(w)-\aleph\left(\neg^{* 1}, \neg^{* 2}, 7^{* 3}, \neg^{* 4}\right)(w)\right| \\
\leq & \varphi\left(\frac{\epsilon}{2}, \frac{\epsilon}{2}, \frac{\epsilon}{2}, \frac{\epsilon}{2}\right) \\
+ & \sigma\left(\vartheta \mid \int_{0}^{\gamma(w)} C\left(w, \partial, \ell^{* 1}(\omega(\partial)), \ell^{* 2}(\omega(\partial)), \ell^{* 3}(\omega(\partial)), \ell^{* 4}(\omega(\partial))\right) d \partial\right.
\end{aligned}
$$




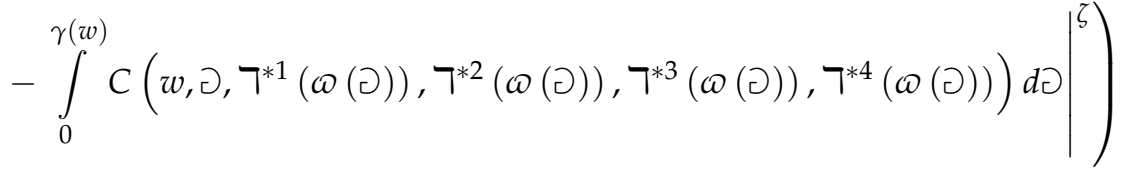

$$
\begin{aligned}
& <\frac{\epsilon}{2}+\sigma\left(\vartheta\left(\gamma^{A} \rho(\epsilon)\right)^{\zeta}\right)
\end{aligned}
$$

where $\gamma^{A}=\sup \{\gamma(w): w \in[0, A]\}$ and:

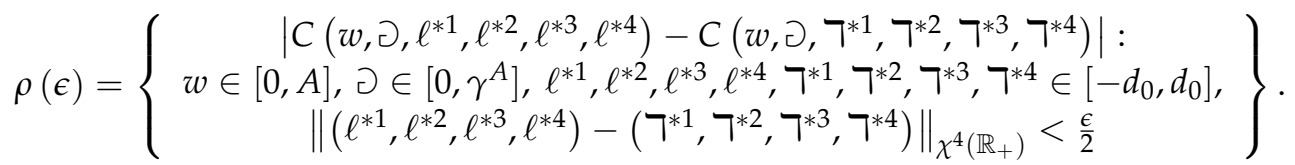

The continuity of $C$ on $[0, A] \times\left[0, \gamma^{A}\right] \times\left[-d_{0}, d_{0}\right]^{4}$ leads to $\lim _{\epsilon \rightarrow 0} \rho(\epsilon)=0$; thus, using the continuity of $\sigma$, we have:

$$
\sigma\left(\vartheta\left(\gamma^{A} \rho(\epsilon)\right)^{\zeta}\right) \rightarrow 0
$$

Therefore, by the two cases, we conclude that $\aleph$ is a continuous function.

Next, we prove that:

$$
\tau_{0}(\aleph(\Lambda)) \leq \varphi\left(\tau_{0}\left(Q^{1}\right), \tau_{0}\left(Q^{2}\right), \tau_{0}\left(Q^{3}\right), \tau_{0}\left(Q^{4}\right)\right),
$$

where $\Lambda=Q^{1} \times Q^{2} \times Q^{3} \times Q^{4}$. For this, let $Q^{1}, Q^{2}, Q^{3}, Q^{4}$ be arbitrary nonempty subsets of $\bar{O}_{d_{0}}$ and $w_{1}, w_{2} \in[0, A]$ with $\left|w_{1}-w_{2}\right| \leq \epsilon$. Without loss of generality, consider $\gamma\left(w_{1}\right) \leq \gamma\left(w_{2}\right)$, and for each $\left(\ell^{* 1}, \ell^{* 2}, \ell^{* 3}, \ell^{* 4}\right) \in \Lambda$, we get:

$$
\begin{aligned}
& \left|\aleph\left(\ell^{* 1}, \ell^{* 2}, \ell^{* 3}, \ell^{* 4}\right)\left(w_{2}\right)-\aleph\left(\ell^{* 1}, \ell^{* 2}, \ell^{* 3}, \ell^{* 4}\right)\left(w_{1}\right)\right| \\
& \leq\left|O\left(w_{2}\right)-O\left(w_{1}\right)\right|+\mid V\left(w_{2}, \ell^{* 1}\left(\delta\left(w_{2}\right)\right), \ell^{* 2}\left(\delta\left(w_{2}\right)\right), \ell^{* 3}\left(\delta\left(w_{2}\right)\right), \ell^{* 4}\left(\delta\left(w_{2}\right)\right)\right) \\
& -V\left(w_{2}, \ell^{* 1}\left(\delta\left(w_{1}\right)\right), \ell^{* 2}\left(\delta\left(w_{1}\right)\right), \ell^{* 3}\left(\delta\left(w_{1}\right)\right), \ell^{* 4}\left(\delta\left(w_{1}\right)\right)\right) \mid \\
& +\mid V\left(w_{2}, \ell^{* 1}\left(\delta\left(w_{1}\right)\right), \ell^{* 2}\left(\delta\left(w_{1}\right)\right), \ell^{* 3}\left(\delta\left(w_{1}\right)\right), \ell^{* 4}\left(\delta\left(w_{1}\right)\right)\right) \\
& -V\left(w_{1}, \ell^{* 1}\left(\delta\left(w_{1}\right)\right), \ell^{* 2}\left(\delta\left(w_{1}\right)\right), \ell^{* 3}\left(\delta\left(w_{1}\right)\right), \ell^{* 4}\left(\delta\left(w_{1}\right)\right)\right) \mid
\end{aligned}
$$

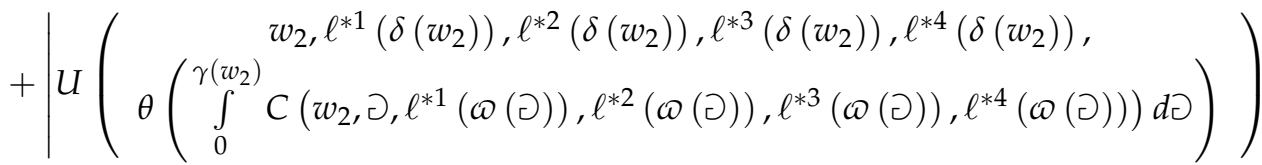

$$
\begin{aligned}
& -U\left(\theta \left(\begin{array}{c}
\gamma\left(w_{2}\right) \\
w_{2}, \ell^{* 1}\left(\delta\left(w_{1}\right)\right), \ell^{* 2}\left(\delta\left(w_{1}\right)\right), \ell^{* 3}\left(\delta\left(w_{1}\right)\right), \ell^{* 4}\left(\delta\left(w_{1}\right)\right), \\
0
\end{array}\right.\right.
\end{aligned}
$$

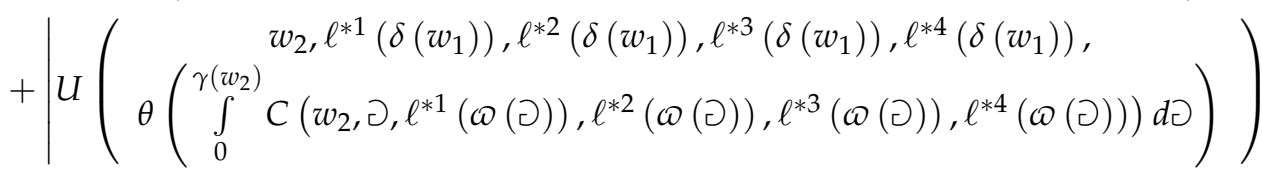

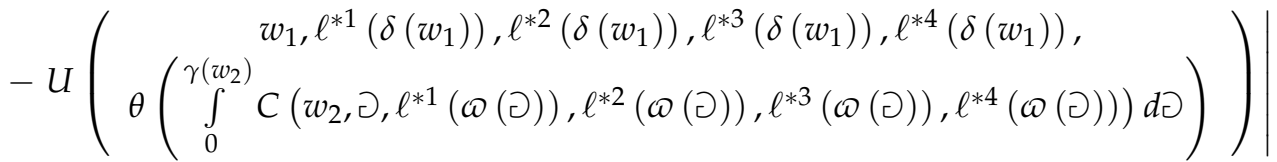




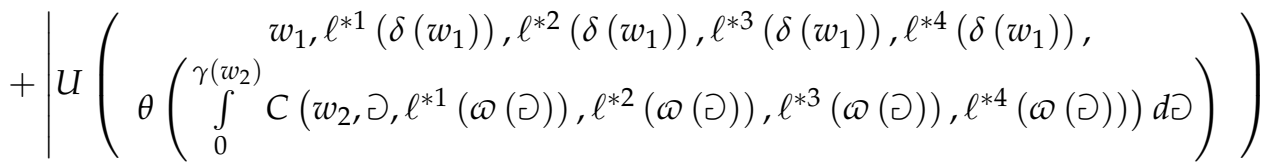

$$
\begin{aligned}
& -U\left(\begin{array}{c}
\gamma\left(w_{2}\right) \\
w_{1}, \ell^{* 1}\left(\delta\left(w_{1}\right)\right), \ell^{* 2}\left(\delta\left(w_{1}\right)\right), \ell^{* 3}\left(\delta\left(w_{1}\right)\right), \ell^{* 4}\left(\delta\left(w_{1}\right)\right), \\
0
\end{array}\right. \\
& +\mid U\left(\theta\left(\begin{array}{c}
\gamma\left(w_{2}\right) \\
\int_{0}, \ell^{* 1}\left(\delta\left(w_{1}\right)\right), \ell^{* 2}\left(\delta\left(w_{1}\right)\right), \ell_{1}^{* 3}\left(\delta, \ell^{* 1}\left(\omega\left(w_{1}\right)\right)\right), \ell^{* 4}\left(\delta\left(w_{1}\right)\right), \\
\left.\left.\ell^{* 2}(\omega(\partial)), \ell^{* 3}(\omega(\circlearrowright)), \ell^{* 4}(\omega(\circlearrowright))\right) d \circlearrowright\right)
\end{array}\right)\right.
\end{aligned}
$$

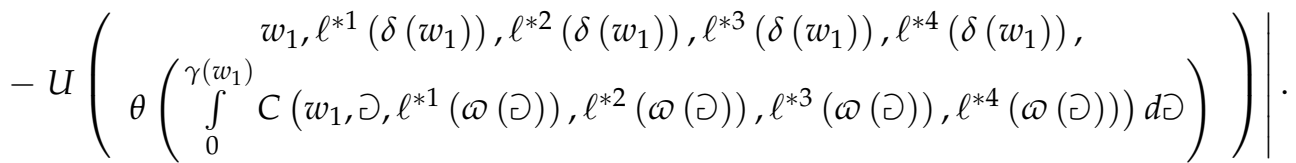

\section{For simplicity, we use the following formulas:}

$$
\begin{aligned}
\tau^{A}(O, \epsilon) & =\sup \left\{\left|O\left(w_{1}\right)-O\left(w_{2}\right)\right|: w_{1}, w_{2} \in[0, A],\left|w_{1}-w_{2}\right| \leq \epsilon\right\}, \\
\tau_{d_{0}}^{A}(V, \epsilon) & =\sup \left\{\begin{array}{c}
\left|V\left(w_{2}, \ell^{* 1}, \ell^{* 2}, \ell^{* 3}, \ell^{* 4}\right)-V\left(w_{1}, \ell^{* 1}, \ell^{* 2}, \ell^{* 3}, \ell^{* 4}\right)\right|: w_{1}, w_{2} \in[0, A], \\
\left|w_{1}-w_{2}\right| \leq \epsilon, \ell^{* 1}, \ell^{* 2}, \ell^{* 3}, \ell^{* 4} \in\left[d_{0}, d_{0}\right]
\end{array}\right\}, \\
\tau^{A}(\delta, \epsilon) & =\sup \left\{\left|\delta\left(w_{1}\right)-\delta\left(w_{2}\right)\right|: w_{1}, w_{2} \in[0, A],\left|w_{1}-w_{2}\right| \leq \epsilon\right\}, \\
\tau^{A}\left(\ell^{* 1}, \tau^{A}(\delta, \epsilon)\right) & =\sup \left\{\left|\ell^{* 1}\left(w_{1}\right)-\ell^{* 1}\left(w_{2}\right)\right|: w_{1}, w_{2} \in[0, A],\left|w_{1}-w_{2}\right| \leq \tau^{A}(\delta, \epsilon)\right\}, \\
\Omega_{d_{0}}^{A} & =\sup \left\{\begin{array}{c}
\left|C\left(w, \partial, \ell^{* 1}, \ell^{* 2}, \ell^{* 3}, \ell^{* 4}\right)\right|: w \in[0, A], \partial \in\left[0, \gamma^{A}\right], \\
\ell^{* 1}, \ell^{* 2}, \ell^{* 3}, \ell^{* 4} \in\left[d_{0}, d_{0}\right]
\end{array}\right\}, \\
\Omega^{*} & =\gamma^{A} \sup \left\{\begin{array}{c}
\left|C\left(w, \partial, \ell^{* 1}, \ell^{* 2}, \ell^{* 3}, \ell^{* 4}\right)\right|: w \in[0, A], \partial \in\left[0, \gamma^{A}\right], \\
\ell^{* 1}, \ell^{* 2}, \ell^{* 3}, \ell^{* 4} \in\left[d_{0}, d_{0}\right]
\end{array}\right\}, \\
\tau_{d_{0}, \Omega^{*}}^{A}(U, \epsilon) & =\sup \left\{\begin{array}{c}
\left|U\left(w_{2}, \ell^{* 1}, \ell^{* 2}, \ell^{* 3}, \ell^{* 4}, \ell^{* 5}\right)-U\left(w_{1}, \ell^{* 1}, \ell^{* 2}, \ell^{* 3}, \ell^{* 4}, \ell^{* 5}\right)\right|: w_{1}, w_{2} \in[0, A], \\
\left|w_{1}-w_{2}\right| \leq \epsilon, \ell^{* 1}, \ell^{* 2}, \ell^{* 3}, \ell^{* 4} \in\left[d_{0}, d_{0}\right], \ell^{* 5} \in\left[-\Omega^{*}, \Omega^{*}\right]
\end{array}\right\}, \\
\tau_{d_{0}}^{A}(C, \epsilon) & =\sup \left\{\begin{array}{c}
\left|C\left(w_{1}, \partial, \ell^{* 1}, \ell^{* 2}, \ell^{* 3}, \ell^{* 4}\right)-C\left(w_{2}, \partial, \ell^{* 1}, \ell^{* 2}, \ell^{* 3}, \ell^{* 4}\right)\right|: w \in[0, A], \\
\partial \in\left[0, \gamma^{A}\right], \ell^{* 1}, \ell^{* 2}, \ell^{* 3}, \ell^{* 4} \in\left[d_{0}, d_{0}\right]
\end{array}\right\}, \\
\tau^{A}(\gamma, \epsilon) & =\sup \left\{\left|\gamma\left(w_{1}\right)-\gamma\left(w_{2}\right)\right|: w_{1}, w_{2} \in[0, A],\left|w_{1}-w_{2}\right| \leq \epsilon\right\} .
\end{aligned}
$$

It follows from (16) and (17) that:

$$
\begin{aligned}
& \left|\aleph\left(\ell^{* 1}, \ell^{* 2}, \ell^{* 3}, \ell^{* 4}\right)\left(w_{2}\right)-\aleph\left(\ell^{* 1}, \ell^{* 2}, \ell^{* 3}, \ell^{* 4}\right)\left(w_{1}\right)\right| \\
& \leq \tau^{A}(O, \epsilon)+\frac{1}{2} \varphi\left(\begin{array}{c}
\left|\ell^{* 1}\left(\delta\left(w_{2}\right)\right)-\ell^{* 1}\left(\delta\left(w_{1}\right)\right)\right|,\left|\ell^{* 2}\left(\delta\left(w_{2}\right)\right)-\ell^{* 2}\left(\delta\left(w_{1}\right)\right)\right|, \\
\left|\ell^{* 3}\left(\delta\left(w_{2}\right)\right)-\ell^{* 3}\left(\delta\left(w_{1}\right)\right)\right|,\left|\ell^{* 4}\left(\delta\left(w_{2}\right)\right)-\ell^{* 4}\left(\delta\left(w_{1}\right)\right)\right|
\end{array}\right) \\
& +\tau_{d_{0}}^{A}(V, \epsilon)+\frac{1}{2} \varphi\left(\begin{array}{c}
\left|\ell^{* 1}\left(\delta\left(w_{2}\right)\right)-\ell^{* 1}\left(\delta\left(w_{1}\right)\right)\right|,\left|\ell^{* 2}\left(\delta\left(w_{2}\right)\right)-\ell^{* 2}\left(\delta\left(w_{1}\right)\right)\right|, \\
\left|\ell^{* 3}\left(\delta\left(w_{2}\right)\right)-\ell^{* 3}\left(\delta\left(w_{1}\right)\right)\right|,\left|\ell^{* 4}\left(\delta\left(w_{2}\right)\right)-\ell^{* 4}\left(\delta\left(w_{1}\right)\right)\right|
\end{array}\right) \\
& +\tau_{d_{0}, \Omega^{*}}^{A}(U, \epsilon)+\sigma\left(\mid \theta\left(\int_{0}^{\gamma\left(w_{2}\right)} C\left(w_{2}, \partial, \ell^{* 1}(\omega(\partial)), \ell^{* 2}(\omega(\partial)), \ell^{* 3}(\omega(\partial)), \ell^{* 4}(\omega(\partial))\right) d \partial\right)\right. \\
& \left.-\theta\left(\int_{0}^{\gamma\left(w_{2}\right)} C\left(w_{1}, \partial, \ell^{* 1}(\omega(\partial)), \ell^{* 2}(\omega(\partial)), \ell^{* 3}(\omega(\partial)), \ell^{* 4}(\omega(\partial))\right) d \partial\right) \mid\right)
\end{aligned}
$$




$$
\begin{aligned}
& +\sigma\left(\mid \theta\left(\int_{0}^{\gamma\left(w_{2}\right)} C\left(w_{1}, \partial, \ell^{* 1}(\omega(\partial)), \ell^{* 2}(\omega(\circlearrowright)), \ell^{* 3}(\omega(\partial)), \ell^{* 4}(\omega(\partial))\right) d \circlearrowright\right)\right. \\
& \left.-\theta\left(\int_{0}^{\gamma\left(w_{1}\right)} C\left(w_{1}, \partial, \ell^{* 1}(\omega(\partial)), \ell^{* 2}(\omega(\partial)), \ell^{* 3}(\omega(\partial)), \ell^{* 4}(\omega(\partial))\right) d \partial\right) \mid\right) \\
& \leq \tau^{A}(O, \epsilon)+\tau_{d_{0}}^{A}(V, \epsilon)+\varphi\left(\begin{array}{c}
\tau^{A}\left(\ell^{* 1}, \tau^{A}(\delta, \epsilon)\right), \tau^{A}\left(\ell^{* 2}, \tau^{A}(\delta, \epsilon)\right), \\
\tau^{A}\left(\ell^{* 3}, \tau^{A}(\delta, \epsilon)\right), \tau^{A}\left(\ell^{* 4}, \tau^{A}(\delta, \epsilon)\right)
\end{array}\right)+\tau_{d_{0}, \Omega^{*}}^{A}(U, \epsilon) \\
& +\sigma\left(\vartheta \mid \int_{0}^{\gamma\left(w_{2}\right)} C\left(w_{2}, \partial, \ell^{* 1}(\omega(\partial)), \ell^{* 2}(\omega(\partial)), \ell^{* 3}(\omega(\partial)), \ell^{* 4}(\omega(\partial))\right)\right. \\
& \left.-\left.C\left(w_{1}, \partial, \ell^{* 1}(\omega(\partial)), \ell^{* 2}(\omega(\partial)), \ell^{* 3}(\omega(\partial)), \ell^{* 4}(\omega(\partial))\right) d \partial\right|^{\zeta}\right) \\
& +\sigma\left(\vartheta\left|\int_{\gamma\left(w_{1}\right)}^{\gamma\left(w_{2}\right)} C\left(w_{1}, \partial, \ell^{* 1}(\omega(\partial)), \ell^{* 2}(\omega(\partial)), \ell^{* 3}(\omega(\partial)), \ell^{* 4}(\omega(\partial))\right) d \partial\right|^{\zeta}\right) \\
& \leq \tau^{A}(O, \epsilon)+\tau_{d_{0}}^{A}(V, \epsilon)+\varphi\left(\begin{array}{c}
\tau^{A}\left(\ell^{* 1}, \tau^{A}(\delta, \epsilon)\right), \tau^{A}\left(\ell^{* 2}, \tau^{A}(\delta, \epsilon)\right), \\
\tau^{A}\left(\ell^{* 3}, \tau^{A}(\delta, \epsilon)\right), \tau^{A}\left(\ell^{* 4}, \tau^{A}(\delta, \epsilon)\right)
\end{array}\right)+\tau_{d_{0,}, \Omega^{*}}^{A}(U, \epsilon) \\
& +\sigma\left(\vartheta\left(\Omega_{d_{0}}^{A} \tau^{A}(\gamma, \epsilon)\right)^{\zeta}\right)+\sigma\left(\vartheta\left(\gamma^{A} \tau_{d_{0}}^{A}(C, \epsilon)\right)^{\zeta}\right) .
\end{aligned}
$$

Since $\left(\ell^{* 1}, \ell^{* 2}, \ell^{* 3}, \ell^{* 4}\right) \in \Lambda$ is arbitrary, then we have:

$$
\begin{aligned}
& \tau^{A}(\aleph(\Lambda), \epsilon) \\
\leq & \tau^{A}(O, \epsilon)+\tau_{d_{0}}^{A}(V, \epsilon)+\varphi\left(\begin{array}{c}
\tau^{A}\left(Q^{1}, \tau^{A}(\delta, \epsilon)\right), \tau^{A}\left(Q^{2}, \tau^{A}(\delta, \epsilon)\right), \\
\tau^{A}\left(Q^{3}, \tau^{A}(\delta, \epsilon)\right), \tau^{A}\left(Q^{4}, \tau^{A}(\delta, \epsilon)\right)
\end{array}\right) \\
& +\tau_{d_{0}, \Omega^{*}}^{A}(U, \epsilon)+\sigma\left(\vartheta\left(\Omega_{d_{0}}^{A} \tau^{A}(\gamma, \epsilon)\right)^{\zeta}\right)+\sigma\left(\vartheta\left(\gamma^{A} \tau_{d_{0}}^{A}(C, \epsilon)\right)^{\zeta}\right) .
\end{aligned}
$$

Since $U, C$ and $V$ are uniform continuous on the compact sets $[0, A] \times\left[-d_{0}, d_{0}\right]^{4} \times\left[-\Omega^{*}, \Omega^{*}\right],[0, A] \times$ $\left[0, \gamma^{A}\right] \times\left[-d_{0}, d_{0}\right]^{4}$, and $[0, A] \times\left[-d_{0}, d_{0}\right]^{4}$, respectively, we have:

$$
\tau_{d_{0}, \Omega^{*}}^{A}(U, \epsilon) \rightarrow 0, \tau_{d_{0}}^{A}(C, \epsilon) \rightarrow 0 \text { and } \tau_{d_{0}, \Omega^{*}}^{A}(V, \epsilon) \rightarrow 0 \text {, as } \epsilon \rightarrow 0 .
$$

Furthermore, the uniform continuity of $\delta, \gamma$, and $O$ on $[0, A]$ leads to:

$$
\tau^{A}(\delta, \epsilon) \rightarrow 0, \tau^{A}(\gamma, \epsilon) \rightarrow 0 \text { and } \tau^{A}(O, \epsilon) \rightarrow 0, \text { as } \epsilon \rightarrow 0 .
$$

Additionally, by the definition of $\sigma$ with $\sigma(0)=0$ and $\Omega^{*}$ finite, we get:

$$
\sigma\left(\vartheta\left(\Omega_{d_{0}}^{A} \tau^{A}(\gamma, \epsilon)\right)^{\zeta}\right)+\sigma\left(\vartheta\left(\gamma^{A} \tau_{d_{0}}^{A}(C, \epsilon)\right)^{\zeta}\right) \rightarrow 0, \text { as } \epsilon \rightarrow 0
$$

Hence, passing the limit as $\epsilon \rightarrow 0$ in (19), one gets:

$$
\tau^{A}(\aleph(\Lambda)) \leq \varphi\left(\tau_{0}^{A}\left(Q^{1}\right), \tau_{0}^{A}\left(Q^{2}\right), \tau_{0}^{A}\left(Q^{3}\right), \tau_{0}^{A}\left(Q^{4}\right)\right) .
$$


When $A \rightarrow \infty$ in (19), we can write:

$$
\tau_{0}(\aleph(\Lambda)) \leq \varphi\left(\tau_{0}\left(Q^{1}\right), \tau_{0}\left(Q^{2}\right), \tau_{0}\left(Q^{3}\right), \tau_{0}\left(Q^{4}\right)\right) .
$$

Finally, we prove that the stipulation (7) of Corollary 1 is fulfilled. Consider $\left(\ell^{* 1}, \ell^{* 2}, \ell^{* 3}, \ell^{* 4}\right)$, (\urcorner$\left.^{* 1}, \boldsymbol{\top}^{* 2}, \boldsymbol{\top}^{* 3}, \boldsymbol{\top}^{* 4}\right) \in \Lambda$ to be arbitrary elements and $w \in \mathbb{R}_{+}$, then by (13), we have:

$$
\begin{aligned}
& \left.\left.\left.\left.\mid \aleph\left(\ell^{* 1}, \ell^{* 2}, \ell^{* 3}, \ell^{* 4}\right)(w)-\aleph(\urcorner^{* 1},\right\rceil^{* 2},\right\urcorner^{* 3},\right\urcorner^{* 4}\right)(w) \mid \\
& \leq \varphi\left(\begin{array}{c}
\left.\left.\mid \ell^{* 1}(\delta(w))-\right\rceil^{* 1}(\delta(w))|,| \ell^{* 2}(\delta(w))-\right\rceil^{* 2}(\delta(w)) \mid, \\
\left.\left|\ell^{* 3}(\delta(w))-T^{* 3}(\delta(w))\right|, \mid \ell^{* 4}(\delta(w))-\right\rceil^{* 4}(\delta(w)) \mid
\end{array}\right) \\
& +\sigma\left(\mid \theta\left(\int_{0}^{\gamma(w)} C\left(w, \partial, \ell^{* 1}(\omega(\partial)), \ell^{* 2}(\omega(\partial)), \ell^{* 3}(\omega(\partial)), \ell^{* 4}(\omega(\partial))\right) d \partial\right)\right. \\
& \left.\left.\left.\left.\left.-\theta \int_{0}^{\gamma(w)} C(w, x,\rceil^{* 1}(\omega(\partial)),\right\rceil^{* 2}(\omega(\partial)),\right\rceil^{* 3}(\omega(\partial)),\right\rceil^{* 4}(\omega(\partial))\right) d \circlearrowright \mid\right) \\
& \leq \varphi\left(\operatorname{diam} Q^{1}(\delta(w)), \operatorname{diam} Q^{2}(\delta(w)), \operatorname{diam} Q^{3}(\delta(w)), \operatorname{diam} Q^{4}(\delta(w))\right) \\
& +\sigma\left(\vartheta \mid \int_{0}^{\gamma(w)} C\left(w, \partial, \ell^{* 1}(\omega(\partial)), \ell^{* 2}(\omega(\partial)), \ell^{* 3}(\omega(\partial)), \ell^{* 4}(\omega(\partial))\right) d \partial\right.
\end{aligned}
$$

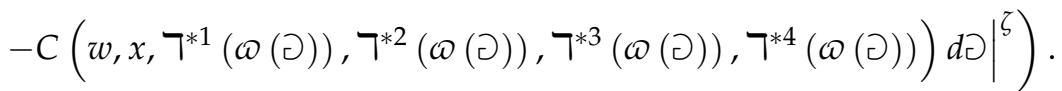

This implies that:

$$
\begin{aligned}
& \operatorname{diam} \aleph(\Lambda)(w) \\
& \leq \varphi\left(\operatorname{diam} Q^{1}(\delta(w)), \operatorname{diam} Q^{2}(\delta(w)), \operatorname{diam} Q^{3}(\delta(w)), \operatorname{diam} Q^{4}(\delta(w))\right) \\
& +\sigma\left(\vartheta \mid \int_{0}^{\gamma(w)} C\left(w, \partial, \ell^{* 1}(\omega(\circlearrowright)), \ell^{* 2}(\omega(\circlearrowright)), \ell^{* 3}(\omega(\circlearrowright)), \ell^{* 4}(\omega(\circlearrowright))\right)\right.
\end{aligned}
$$

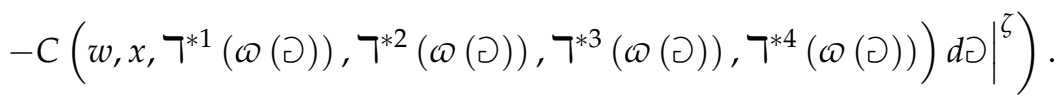

As $w \rightarrow \infty$ in (22) and by (11), we have:

$$
\lim \sup _{w \rightarrow \infty} \operatorname{diam} \aleph(\Lambda)(w) \leq \varphi\left(\begin{array}{l}
\lim \sup _{w \rightarrow \infty} \operatorname{diam} Q^{1}(\delta(w)), \\
\lim \sup _{w \rightarrow \infty} \operatorname{diam} Q^{2}(\delta(w)), \\
\lim \sup _{w \rightarrow \infty} \operatorname{diam} Q^{3}(\delta(w)), \\
\lim \sup _{w \rightarrow \infty} \operatorname{diam} Q^{4}(\delta(w))
\end{array}\right)
$$

It follows from (20) and (22) that:

$$
\begin{array}{r}
\tau_{0}(\aleph(\Lambda)) \leq \varphi\left(\tau_{0}\left(Q^{1}\right), \tau_{0}\left(Q^{2}\right), \tau_{0}\left(Q^{3}\right), \tau_{0}\left(Q^{4}\right)\right) \\
+\varphi\left(\begin{array}{l}
\lim \sup _{w \rightarrow+\infty} \operatorname{diam} Q^{1}(\delta(w)), \\
\lim \sup _{w \rightarrow+\infty} \operatorname{diam} Q^{2}(\delta(w)), \\
\lim \sup _{w \rightarrow+\infty} \operatorname{diam} Q^{3}(\delta(w)), \\
\lim \sup _{w \rightarrow+\infty} \operatorname{diam} Q^{4}(\delta(w))
\end{array}\right) .
\end{array}
$$


Now, since $\varphi$ is continuous, non-negative, and $\varphi(\ell, \ell, \ell, \ell)<\ell$ by property $\Re_{2}$, it must hold that $\varphi(0,0,0,0)=0$. Thus:

$$
\begin{array}{r}
\tau_{0}(\aleph(\Lambda)) \leq \quad \varphi\left(\tau_{0}\left(Q^{1}\right), \tau_{0}\left(Q^{2}\right), \tau_{0}\left(Q^{3}\right), \tau_{0}\left(Q^{4}\right)\right) \\
+\varphi\left(\begin{array}{l}
\lim \sup _{w \rightarrow+\infty} \operatorname{diam} Q^{1}(\delta(w)), \\
\limsup _{w \rightarrow+\infty} \operatorname{diam} Q^{2}(\delta(w)), \\
\limsup _{w \rightarrow+\infty} \operatorname{diam} Q^{3}(\delta(w)), \\
\limsup \sup _{w \rightarrow+\infty} \operatorname{diam} Q^{4}(\delta(w))
\end{array}\right) \\
\leq 4 \varphi\left(\begin{array}{c}
\frac{\tau_{0}\left(Q^{1}\right)+\limsup \sup _{w \rightarrow+\infty} \operatorname{diam} Q^{1}(\delta(w))}{4} \operatorname{diam} Q^{2}(\delta(w)) \\
\frac{\tau_{0}\left(Q^{2}\right)+\limsup \sup _{w \rightarrow+\infty} \operatorname{diam} Q^{3}(\delta(w))}{4}, \\
\frac{\tau_{0}\left(Q^{3}\right)+\limsup \sup _{w \rightarrow+\infty} \operatorname{dia}\left(Q^{4}\right)+\lim \sup _{w \rightarrow+\infty} \operatorname{diam} Q^{4}(\delta(w))}{4}
\end{array}\right) .
\end{array}
$$

Now, define $\mho=\frac{\tau_{0}}{4}$, which is still an MNC, and by (9), we get:

$$
\mho(\aleph(\Lambda)) \leq \varphi\left(\mho\left(Q^{1}\right), \mho\left(Q^{2}\right), \mho\left(Q^{3}\right), \mho\left(Q^{4}\right)\right) .
$$

Therefore, by Corollary $1, \aleph$ has at least one QFP in $\chi^{4}\left(\mathbb{R}_{+}\right)$. This ends the proof.

\section{Illustrative Example}

In this part, we introduce the example below.

Example 4. Consider the following system:

$$
\begin{aligned}
& \ell^{* 1}(w)=\left(\frac{1}{4\left(1+w^{2}\right)}+\frac{w^{4}}{3\left(1+w^{4}\right)}+\frac{1}{2\left(1+e^{w}\right)}\right)+\left(\frac{e^{w}}{10\left(1+e^{w}\right)}+\frac{e^{2 w}}{12\left(1+e^{2 w}\right)}\right) \ell^{* 1}(w) \\
& +\left(\frac{e^{w^{2}}}{20\left(1+e^{w^{2}}\right)}+\frac{1}{24}\right) \ell^{* 2}(w)+\left(\frac{e^{w^{3}}}{30\left(1+e^{w^{3}}\right)}+\frac{w^{2}}{36\left(1+w^{2}\right)}\right) \ell^{* 3}(w) \\
& +\left(\frac{e^{w^{4}}}{40\left(1+e^{w^{4}}\right)}+\frac{1}{48}\right) \ell^{* 4}(w) \\
& +\int_{0}^{w} \frac{\partial\left|\cos \ell^{* 1}(w)\right|\left|\cos \ell^{* 2}(w)\right|\left|\cos \ell^{* 3}(w)\right|\left|\cos \ell^{* 4}(w)\right|}{e^{w}\left(1+\cos ^{2} \ell^{* 1}(w)\right)\left(1+\cos ^{2} \ell^{* 2}(w)\right)\left(1+\cos ^{2} \ell^{* 3}(w)\right)\left(1+\cos ^{2} \ell^{* 4}(w)\right)} d \partial, \\
& \ell^{* 2}(w)=\left(\frac{1}{4\left(1+w^{2}\right)}+\frac{w^{4}}{3\left(1+w^{4}\right)}+\frac{1}{2\left(1+e^{w}\right)}\right)+\left(\frac{e^{w}}{10\left(1+e^{w}\right)}+\frac{e^{2 w}}{12\left(1+e^{2 w}\right)}\right) \ell^{* 2}(w) \\
& +\left(\frac{e^{w^{2}}}{20\left(1+e^{w^{2}}\right)}+\frac{1}{24}\right) \ell^{* 3}(w)+\left(\frac{e^{w^{3}}}{30\left(1+e^{w^{3}}\right)}+\frac{w^{2}}{36\left(1+w^{2}\right)}\right) \ell^{* 4}(w) \\
& +\left(\frac{e^{w^{4}}}{40\left(1+e^{w^{4}}\right)}+\frac{1}{48}\right) \ell^{* 1}(w) \\
& +\int_{0}^{w} \frac{\partial\left|\cos \ell^{* 1}(w)\right|\left|\cos \ell^{* 2}(w)\right|\left|\cos \ell^{* 3}(w)\right|\left|\cos \ell^{* 4}(w)\right|}{e^{w}\left(1+\cos ^{2} \ell^{* 2}(w)\right)\left(1+\cos ^{2} \ell^{* 3}(w)\right)\left(1+\cos ^{2} \ell^{* 4}(w)\right)\left(1+\cos ^{2} \ell^{* 1}(w)\right)} d \circlearrowright, \\
& \ell^{* 3}(w)=\left(\frac{1}{4\left(1+w^{2}\right)}+\frac{w^{4}}{3\left(1+w^{4}\right)}+\frac{1}{2\left(1+e^{w}\right)}\right)+\left(\frac{e^{w}}{10\left(1+e^{w}\right)}+\frac{e^{2 w}}{12\left(1+e^{2 w}\right)}\right) \ell^{* 3}(w) \\
& +\left(\frac{e^{w^{2}}}{20\left(1+e^{w^{2}}\right)}+\frac{1}{24}\right) \ell^{* 4}(w)+\left(\frac{e^{z w^{3}}}{30\left(1+e^{w^{3}}\right)}+\frac{w^{2}}{36\left(1+w^{2}\right)}\right) \ell^{* 1}(w)
\end{aligned}
$$




$$
\begin{aligned}
& +\left(\frac{e^{w^{4}}}{40\left(1+e^{w^{4}}\right)}+\frac{1}{48}\right) \ell^{* 2}(w) \\
& +\int_{0}^{w} \frac{\partial\left|\cos \ell^{* 1}(w)\right|\left|\cos \ell^{* 2}(w)\right|\left|\cos \ell^{* 3}(w)\right|\left|\cos \ell^{* 4}(w)\right|}{e^{w}\left(1+\cos ^{2} \ell^{* 3}(w)\right)\left(1+\cos ^{2} \ell^{* 4}(w)\right)\left(1+\cos ^{2} \ell^{* 1}(w)\right)\left(1+\cos ^{2} \ell^{* 2}(w)\right)} d \circlearrowright, \\
\ell^{* 4}(w)= & \left(\frac{1}{4\left(1+w^{2}\right)}+\frac{w^{4}}{3\left(1+w^{4}\right)}+\frac{1}{2\left(1+e^{w}\right)}\right)+\left(\frac{e^{w}}{10\left(1+e^{w}\right)}+\frac{e^{2 w}}{12\left(1+e^{2 w}\right)}\right) \ell^{* 4}(w) \\
& +\left(\frac{e^{w^{2}}}{20\left(1+e^{w^{2}}\right)}+\frac{1}{24}\right) \ell^{* 1}(w)+\left(\frac{e^{w^{3}}}{30\left(1+e^{w^{3}}\right)}+\frac{w^{2}}{36\left(1+w^{2}\right)}\right) \ell^{* 2}(w) \\
& +\left(\frac{e^{w^{4}}}{40\left(1+e^{w^{4}}\right)}+\frac{1}{48}\right) \ell^{* 3}(w) \\
& +\int_{0}^{w} \frac{\partial\left|\cos \ell^{* 1}(w)\right|\left|\cos \ell^{* 2}(w)\right|\left|\cos \ell^{* 3}(w)\right|\left|\cos \ell^{* 4}(w)\right|}{e^{w}\left(1+\cos ^{2} \ell^{* 4}(w)\right)\left(1+\cos ^{2} \ell^{* 1}(w)\right)\left(1+\cos ^{2} \ell^{* 2}(w)\right)\left(1+\cos ^{2} \ell^{* 3}(w)\right)} d \circlearrowright .
\end{aligned}
$$

It is obvious that the system (23) is a special form of the system (8) with the following:

- $O(w)=\frac{1}{4\left(1+w^{2}\right)}$,

- $\quad V\left(w, \ell^{* 1}, \ell^{* 2}, \ell^{* 3}, \ell^{* 4}\right)=\frac{w^{4}}{3\left(1+w^{4}\right)}+\frac{e^{w^{w}}}{10\left(1+e^{w}\right)} \ell^{* 1}+\frac{e^{w^{2}}}{20\left(1+e^{w^{2}}\right)} \ell^{* 2}+\frac{e^{w^{3}}}{30\left(1+e^{w^{3}}\right)} \ell^{* 3}+\frac{e^{w^{4}}}{40\left(1+e^{w^{4}}\right)} \ell^{* 4}$,

- $U\left(w, \ell^{* 1}, \ell^{* 2}, \ell^{* 3}, \ell^{* 4}, D\right)=\frac{1}{2\left(1+e^{w}\right)}+\frac{e^{2 w}}{12\left(1+e^{2 w}\right)} \ell^{* 1}+\frac{1}{24} \ell^{* 2}+\frac{w^{2}}{36\left(1+w^{2}\right)} \ell^{* 3}+\frac{1}{48} \ell^{* 4}+D$,

- $C\left(w, \circlearrowright, \ell^{* 1}, \ell^{* 2}, \ell^{* 3}, \ell^{* 4}\right)=\frac{\partial\left|\cos \ell^{* 1}(w)\right|\left|\cos \ell^{* 2}(w)\right|\left|\cos \ell^{* 3}(w)\right|\left|\cos \ell^{* 4}(w)\right|}{e^{w}\left(1+\cos ^{2} \ell^{* 1}(w)\right)\left(1+\cos ^{2} \ell^{* 2}(w)\right)\left(1+\cos ^{2} \ell^{* 3}(w)\right)\left(1+\cos ^{2} \ell^{* 4}(w)\right)}$,

- $\delta(w)=\omega(w)=\gamma(w)=\sigma(w)=\theta(w)=w$,

- $\varphi(w, \partial, \ell\urcorner,)=\frac{w+\partial+\ell+\urcorner}{5}$.

Now, we shall verify the hypotheses of Theorem 7.

- $\quad$ The hypothesis $\left(\nabla_{1}\right)$ is fulfilled, since $O(w)=\frac{1}{4\left(1+w^{2}\right)}$ is continuous on $\mathbb{R}_{+}$and $\xi_{1}=\frac{1}{4}$.

- From the definition of $\delta(w), \omega(w)$ and $\gamma(w)$, we see that $\delta(w)=\omega(w)=\gamma(w)=w$ are continuous and $\delta(w) \rightarrow \infty$ as $w \rightarrow \infty$, so the hypothesis $\left(\nabla_{2}\right)$ is satisfied.

- Since $\theta(w)=w$, for $\vartheta, \zeta=1$, the inequality (10) is verified; hence, $\left(\nabla_{4}\right)$ is as well.

- It is easy to see that $U(w, 0,0,0,0,0)=\frac{1}{2\left(1+e^{w}\right)}, V(w, 0,0,0,0)=\frac{w^{4}}{3\left(1+w^{4}\right)}, \xi_{2}=\frac{1}{2}$, and $\xi_{3}=\frac{1}{3}$.

Furthermore, we can write:

$$
\begin{aligned}
& \left|V\left(w, \ell^{* 1}, \ell^{* 2}, \ell^{* 3}, \ell^{* 4}\right)-V\left(w, \boldsymbol{7}^{* 1}, \boldsymbol{\top}^{* 2}, \boldsymbol{\top}^{* 3}, \boldsymbol{\top}^{* 4}\right)\right| \\
& \leq\left|\frac{e^{w}}{10\left(1+e^{w}\right)}\right|\left|\ell^{* 1}-T^{* 1}\right|+\left|\frac{e^{w^{2}}}{20\left(1+e^{w^{2}}\right)}\right|\left|\ell^{* 2}-T^{* 2}\right| \\
& +\left|\frac{e^{w^{3}}}{30\left(1+e^{w^{3}}\right)}\right|\left|\ell^{* 3}-7^{* 3}\right|+\left|\frac{e^{w^{4}}}{40\left(1+e^{w^{4}}\right)}\right|\left|\ell^{* 4}-7^{* 4}\right| \\
& \left.\leq \frac{1}{10} \mid \ell^{* 1}-\right\urcorner^{* 1}\left|+\frac{1}{20}\right| \ell^{* 2}-\nearrow^{* 2}\left|+\frac{1}{30}\right| \ell^{* 3}-\nearrow^{* 3}\left|+\frac{1}{40}\right| \ell^{* 4}-\nearrow^{* 4} \mid \\
& \leq \frac{1}{2}\left(\frac{1}{5}\left|\ell^{* 1}-\nearrow^{* 1}\right|+\frac{1}{5}\left|\ell^{* 2}-7^{* 2}\right|+\frac{1}{5}\left|\ell^{* 3}-7^{* 3}\right|+\frac{1}{5}\left|\ell^{* 4}-7^{* 4}\right|\right) \\
& \leq \frac{1}{2} \varphi\left(\left|\ell^{* 1}-\nearrow^{* 1}\right|,\left|\ell^{* 2}-\nearrow^{* 2}\right|,\left|\ell^{* 3}-\nearrow^{* 3}\right|+\left|\ell^{* 4}-\nearrow^{* 4}\right|\right) \text {; }
\end{aligned}
$$

similarly,

$$
\left.\left.\left.\left.\mid U\left(w, \ell^{* 1}, \ell^{* 2}, \ell^{* 3}, \ell^{* 4}, D_{1}\right)-U(w,\urcorner^{* 1},\right\urcorner^{* 2},\right\urcorner^{* 3},\right\urcorner^{* 4}, D_{2}\right) \mid
$$




$$
\begin{aligned}
& \leq\left|\frac{e^{2 w}}{12\left(1+e^{2 w}\right)}\right|\left|\ell^{* 1}-\neg^{* 1}\right|+\frac{1}{24}\left|\ell^{* 2}-\neg^{* 2}\right| \\
& +\left|\frac{w^{2}}{36\left(1+w^{2}\right)}\right|\left|\ell^{* 3}-7^{* 3}\right|+\frac{1}{48}\left|\ell^{* 4}-7^{* 4}\right|+\left|D_{1}-D_{2}\right| \\
& \left.\left.\leq \frac{1}{12} \mid \ell^{* 1}-\right\urcorner^{* 1}\left|+\frac{1}{24}\right| \ell^{* 2}-\nearrow^{* 2}\left|+\frac{1}{36}\right| \ell^{* 3}-\right\urcorner^{* 3}\left|+\frac{1}{48}\right| \ell^{* 4}-7^{* 4}|+| D_{1}-D_{2} \mid \\
& \left.\left.\leq \frac{1}{2}\left(\frac{1}{5}\left|\ell^{* 1}-\neg^{* 1}\right|+\frac{1}{5}\left|\ell^{* 2}-\neg^{* 2}\right|+\frac{1}{5} \mid \ell^{* 3}-\right\urcorner^{* 3}\left|+\frac{1}{5}\right| \ell^{* 4}-\right\urcorner^{* 4} \mid\right)+\left|D_{1}-D_{2}\right| \\
& \leq \frac{1}{2} \varphi\left(\left|\ell^{* 1}-\boldsymbol{\top}^{* 1}\right|,\left|\ell^{* 2}-\boldsymbol{T}^{* 2}\right|,\left|\ell^{* 3}-\boldsymbol{\top}^{* 3}\right|+\left|\ell^{* 4}-\boldsymbol{\top}^{* 4}\right|\right)+\sigma\left(\left|D_{1}-D_{2}\right|\right) \text {. }
\end{aligned}
$$

Thus, we find that the hypotheses $\left(\nabla_{3}\right)$ and $\left(\nabla_{5}\right)$ are satisfied.

\section{Conclusions}

In nonlinear functional analysis, measures of noncompactness have many applications, especially in metric and topological fixed point theory. This technique allows us to obtain the existence solution of functional integral equations. In Banach algebras, functional integral equations are considered in complicated form, and a well-developed system is required to study this type of equation. Therefore, in our manuscript, the technique is successfully applied to obtain the solution of these equations in a Banach space. Furthermore, some quadruple fixed points consequences with illustrative examples are derived.

Author Contributions: Conceptualization, H.A.H.; investigation, H.A.H.; methodology, H.A.H.; validation, H.A.H.; writing-the original draft, H.A.H.; funding acquisition, A.A.K.; methodology, A.A.K.; project administration, A.A.K. All authors read and agreed to the published version of the manuscript.

Funding: This work was funded by the Academy of Scientific Research and Technology, Egypt, under Science UP Grant No. 6650.

Acknowledgments: The authors are grateful to the Academy of Scientific Research and Technology for the financial support.

Conflicts of Interest: The authors declare no conflict of interest.

\section{References}

1. Agarwal, R.P.; Hussain, N.; Taoudi, M.A. Fixed point theorems in ordered Banach spaces and applications to nonlinear integral equations. Abstr. Appl. Anal. 2012, 2012, 245872. [CrossRef]

2. Aghajani, A.; Jalilian, Y. Existence and global attractivity of solutions of a nonlinear functional integral equation. Commun. Nonlinear Sci. Numer. Simul. 2010, 15, 3306-3312. [CrossRef]

3. Aghajani, A.; Jalilian, Y. Existence of nondecreasing positive solutions for a system of singular integral equations. Mediterr. J. Math. 2011, 8, 563-576. [CrossRef]

4. Dhage, B.; Bellale, S.S. Local asymptotic stability for nonlinear quadratic functional integral equations. Electron. J. Qual. Theory Differ. Equ. 2008, 10,1-13. [CrossRef]

5. Hammad, H.A.; De la Sen, M. Generalized contractive mappings and related results in $b$-metric like spaces with an application. Symmetry 2019, 11, 667. [CrossRef]

6. Hammad, H.A.; De la Sen, M. A Solution of Fredholm integral equation by using the cyclic $\eta_{s}^{q}$-rational contractive mappings technique in $b$-metric-like spaces. Symmetry 2019, 11, 1184. [CrossRef]

7. Hammad, H.A.; De la Sen, M. Solution of nonlinear integral equation via fixed point of cyclic $\alpha_{s}^{q}$-rational contraction mappings in metric-like spaces. Bull. Braz. Math. Soc. New Ser. 2020, 51, 81-105. [CrossRef]

8. Brouwer, L.E.J. ber abbildung von mannigfaltigkeiten. Math. Ann. 1911, 71, 97-115. [CrossRef]

9. Banach, S. Sur les oprations dans les ensembles abstraits et leur application aux quations intgrales. Fund. Math. 1992, 3, 133-181. [CrossRef]

10. Schauder, J. Der fixpunktsatz in funktionalraumen. Stud. Math. 1930, 2, 171-180. [CrossRef]

11. Kuratowski, C. Sur les espaces complets et rgulirement complets. Proc. Jpn. Acad. 1930, 30, 912-916. 
12. Darbo, G. Punti uniti in trasformazioni a codominio non compatto. Rend. Semin. Mat. Della Univ. Padova $1955,24,84-92$.

13. Aghajani, A.; Bana, J.; Sabzali, N. Some generalizations of Darbo fixed point theorem and applications. Bull. Belg. Math. Soc. 2017, 18, 595. [CrossRef]

14. Aghajani, A.; Haghighi, A.S. Existence of solutions for a system of integral equations via measure of noncompactness. Novi Sad J. Math. 2014, 44, 59-73.

15. Allahyari, R.; Aghajani, A. Some extensions of Darbos theorem and solutions of integral equations of Hammerstein type. Int. J. Nonlinear Anal. Appl. 2017, 8, 337-351.

16. Arab, R. Some generalizations of Darbo fixed point theorem and its application. Miskolc Math. Notes 2017, 18, 595-610. [CrossRef]

17. Cai, L.; Liang, J. New generalizations of Darbos fixed point theorem. Fixed Point Theory Appl. 2015, $2015,156$. [CrossRef]

18. Bansa, J.; Rzepka, B. An application of a measure of noncompactness in the study of asymptotic stability. Appl. Math. Lett. 2003, 16, 1-6. [CrossRef]

19. Aghajani, A.; Allahyari, R.; Mursaleen, M. A generalization of Darbo's theorem with application to the solvability of systems of integral equations. J. Comput. Appl. Math. 2014, 260, 68-77. [CrossRef]

20. Roshan, J.R. Existence of solutions for a class of system of functional integral equation via measure of noncompactness. J. Comput. Appl. Math. 2017, 313, 129-141. [CrossRef]

21. Karakaya, V.; Bouzara, N.E.H.; Doan, K.; Atalan, Y. Existence of tripled fixed points for a class of condensing operators in Banach spaces. Sci. World J. 2014, 2014, 541862. [CrossRef] [PubMed]

22. Bana, J. On measures of noncompactness in Banach spaces. Comment. Math. Univ. Carol. 1980, 21, $131-143$.

23. Kreyszig, E. Introductory Functional Analysis with Applications; Wiley: New York, NY, USA, 1978.

24. Bhaskar, T.G.; Lakshmikantham, V. Fixed point theorems in partially ordered metric spaces and applications. Nonlinear Anal. 2006, 65, 1379-1393. [CrossRef]

25. Abbas, M.; Khan, M.A.; Radenović, S. Common coupled fixed point theorems in cone metric spaces for w-compatible mappings. Appl. Math. Comput. 2010, 217, 195-202. [CrossRef]

26. Aydi, H.; Postolache, M.; Shatanawi, W. Coupled fixed point results for $(\psi, \phi)$-weakly contractive mappings in ordered G-metric spaces. Comput. Math. Appl. 2012, 63, 298-309. [CrossRef]

27. Berinde, V. Coupled fixed point theorems for contractive mixed monotone mappings in partially ordered metric spaces. Nonlinear Anal. 2012, 75, 3218-3228. [CrossRef]

28. Hammad, H.A.; De la Sen, M. A coupled fixed point technique for solving coupled systems of functional and nonlinear integral equations. Mathematics 2019, 7, 634. [CrossRef]

29. Hammad, H.A.; Albaqeri, D.M.; Rashwan, R.A. Coupled coincidence point technique and its application for solving nonlinear integral equations in RPOCbML spaces. J. Egypt. Math. Soc. 2020, 28, 8. [CrossRef]

30. Akhmerov, R.R.; Kamenskii, M.I.; Potapov, A.S.; Rodkina, A.E.; Sadovskii, B.N. Measures of Noncompactness and Condensing Operators; Birkhäuser: Basel, Switzerland, 1992.

31. Berinde, V.; Borcut, M. Tripled fixed point theorems for contractive type mappings in partially ordered metric spaces. Nonlinear Anal. 2011, 74, 4889-4897. [CrossRef]

32. Borcut, M. Tripled coincidence theorems for contractive type mappings in partially ordered metric spaces. Appl. Math. Comput. 2012, 218, 7339-7346.

33. Aydi, H.; Abbas, M.; Sintunavarat, W.; Kumam, P. Tripled fixed point of $W$-compatible mappings in abstract metric spaces. Fixed Point Theory Appl. 2012, 2012, 134. [CrossRef]

34. Hammad, H.A.; De la Sen, M. A technique of tripled coincidence points for solving a system of nonlinear integral equations in POCML spaces. J. Inequal. Appl. 2020, 2020, 211. [CrossRef]

35. Hammad, H.A.; De la Sen, M. A tripled fixed point technique for solving a tripled-system of integral equations and Markov process in CCbMS. Adv. Differ. Equ. 2020, 2020, 567. [CrossRef]

36. Karapinar, E.; Luong, N.V. Quadruple fixed point theorems for nonlinear contractions. Comput. Math. Appl. 2012, 64, 1839-1848. [CrossRef]

37. Karapinar, E.; Shatanawi, W.; Mustafa, Z. Quadruple fixed point theorems under nonlinear contractive conditions in partially ordered metric spaces. J. Appl. Math. 2012, 2012, 951912. [CrossRef]

38. Karapinar, E. Quadruple fixed point theorems for weak $\varphi$-contractions. ISRN Math. Anal. 2011, $2011,989423$. 
39. Karapinar, E.; Berinde, V. Quadruple fixed point theorems for nonlinear contractions in partially ordered metric spaces. Banach J. Math. Anal. 2012, 6, 74-89. [CrossRef]

40. Liu, X.L. Quadruple fixed point theorems in partially ordered metric spaces with mixed $g$-monotone property. Fixed Point Theory Appl. 2013, 2013, 147. [CrossRef]

Publisher's Note: MDPI stays neutral with regard to jurisdictional claims in published maps and institutional affiliations.

(C) 2020 by the authors. Licensee MDPI, Basel, Switzerland. This article is an open access article distributed under the terms and conditions of the Creative Commons Attribution (CC BY) license (http:/ / creativecommons.org/licenses/by/4.0/). 